\title{
A Characterization of "Rapidity" Curve in the Chiral Potts Model
}

\section{Shi-shyr Roan}

Max-Planck-Institut für Mathematik, Gottfried-Claren-Straße 26, W-5300 Bonn 3, Federal Republic of Germany

Received August 12, 1991

Abstract. We study the geometry of high genus curves of rapidity variables in chiral Potts model. In terms of symmetries, we characterize these Rieman surfaces and derive their period matrices. By the theory of prime forms, the temperaturelike parameter is expressed by hyperelliptic theta functions.

\section{Introduction}

Nowadays much attention is paid to the exactly solvable models of statistical mechanics. The integrable $N$-state chiral Potts model is the first solvable model where the Boltzmann weights lie on a complex curve of genus greater than one. It contains the natural generalization of Ising model and many of the remarkable properties known for the Ising model $(N=2)$ can be extended to the cases $N \geqq 3$ (see refs. $[1,5,8,15]$ and references therein). This model gives the solution of YangBaxter equation (or the star-triangle equation) which has a very simple product form for the Boltzmann weights

$$
\begin{aligned}
& \frac{W_{p q}(n)}{W_{p q}(0)}=\prod_{j=1}^{n} \frac{d_{p} b_{q}-a_{p} c_{q} \omega^{j}}{b_{p} d_{q}-c_{p} a_{q} \omega^{j}}, \\
& \frac{\overline{W_{p q}}(n)}{\overline{W_{p q}}(0)}=\prod_{j=1}^{n} \frac{\omega \mathrm{a}_{p} d_{q}-d_{p} a_{q} \omega^{j}}{c_{p} b_{q}-b_{p} c_{q} \omega^{j}},
\end{aligned}
$$

depending on the two "rapidities" $p, q$ and a parameter $k^{\prime}$, where

$$
\omega=e^{\frac{2 \pi i}{N}}, \quad j: \text { integer mod. } N \text {. }
$$

The "rapidity" variables $p, q$ are represented by the ratio of 4-vectors $[a, b, c, d]$ satisfying

$$
\begin{aligned}
& k a^{N}+k^{\prime} c^{N}=d^{N}, \\
& k b^{N}+k^{\prime} d^{N}=c^{N},
\end{aligned}
$$


with

$$
k^{2}+k^{\prime 2}=1, \quad k^{\prime 2} \neq 0,1
$$

These equations describe a complex curve of genus $N^{3}-2 N^{2}+1$ as the intersection of two "Fermat surfaces" in $\mathbb{P}^{3}$, or as the fiber product of two Fermat curves over the rational curve. It has a group of automorphisms of order $4 N^{3}$ and the quotient of some $N^{2}$ automorphisms gives a genus $(N-1)$ hyperelliptic curve which is represented by

$$
W_{N, k^{\prime}}: t^{N}=\frac{\left(1-k^{\prime} \lambda\right)\left(1-k^{\prime} \lambda^{-1}\right)}{k^{2}}, \quad(t, \lambda) \in \mathbb{C}^{2} .
$$

(The integer $N$ shall always be assumed to be $\geqq 3$ unless otherwise specified.) The transfer matrix $T(u)$ of the classical statistical mechanical model now depends on the variables lying on Riemann surface $W_{N, k^{\prime}}$, and it satisfies the commutating relation for different $u, u^{\prime}$. However this is not sufficient to solve the eigenvalue problem of the quantum chiral Potts spin chain associated to $T(u)$. One needs a functional equation for the transfer matrix $T(u)$ which was obtained by $[1,2,11$, 13]. For the superintegrable case, when the "vertical rapidity" $p$ has a special value, some simplifications occur and the eigenvalue problem for $T(u)$ are solved in $[1,2,9]$. For the general case, one can explicitly solve the functional equation for the largest eigenvalue in the large-lattice limit $[10,11]$, and compute the spectrum of low-lying excitations to discuss the phenomena of level crossing transition to a new ground state [16] from the physical consideration. Here no uniformizing substitutions are used to obtain all those results. It is the belief that these results should be Abelian integrals over the Riemann surface. One difficulty is that it lacks the "different property" as in the eight-vertex model solved by Baxter, where the uniformizing parametrization leads to the elliptic functions. However in $[6,7]$, the significance of the Abelian functions was indicated in the computation of single-spin expectation values of the general chiral Potts $N$-state model. Hence the theory of prime form of hyperelliptic curves [17] is expected to immerse into the computation of the interesting physical quantities.

This note deals with two mathematical problems arisen from the above rational family $W_{N, k^{\prime}}$ of hyperelliptic curves. In this paper, we shall call $W_{N, k^{\prime}}$ a chiral Potts $N$-state curve, or simply a CP $N$-curve. The first problem is to give a geometrical characterization of this family of CP $N$-curves among the hyperelliptic curves and compare them with the case of elliptic curves. The other is to describe the relationship between the symmetries of a CP $N$-curve and its Jacobian, in particular how the general prime form of Riemann surfaces can be applied to the expression of $k^{\prime}$ in terms of the period of $W_{N, k^{\prime}}$ which was obtained by Baxter in [6].

First we note that $W_{N, k^{\prime}}$ for $N=2$ is the algebraic form of an elliptic curve with a spin 1/2 structure. As an 1-dimension complex torus, the fixed part of the canonical involution $\theta$ of an elliptic curve $E$ consists of 4 elements. It is in one-one correspondence with the branched loci $S$ under the natural projection from $E$ to $\mathbb{P}^{1}(=E /\langle\theta\rangle)$. The order 2 translation of $E$ corresponding to the spin $1 / 2$ structure induces an automorphism of $\mathbb{P}^{1}$, and its orbits in $S$ form a partition of it with each member having 2 elements. Such partition of $S$ naturally leads to the algebraic expression of $W_{N, k^{\prime}}$ for $N=2$, also in the same process to the general $N \geqq 3$. The original Boltzmann weights $a, b, c, d$ are closely connected to the elements in $S$. The derivation and their relationship will be given in Sect. 2 and 4. In Sect. 1, we 
discuss the parametrization of these partitions of $S$ for a subset $S$ of $\mathbb{P}^{1}$ with 4 elements. The formulation is guided by the intent of finding the geometrical meaning of the relations between different variables which appeared in the physical literatures $[1,6,10]$. In Sect. 3 , we shall derive the original "product Fermat" curve of the variable $[a, b, c, d]$ from the curve $W_{N, k^{\prime}}$ and the other two related ones, $W_{N, i k^{\prime} / k}, W_{N, k}$. In Sect. 5, we shall discuss the uniformizing parametrization of a chiral Potts $N$-state curve. The special form of the period obtained by Baxter [6] is discussed from the geometrical point of view, that is how does it reflect the symmetries of the Riemann surface. We apply the theory of prime form to obtain the explicit expression of the parameter $k^{\prime}$ in terms of the hyperelliptic theta functions.

I am much indebted to Prof. B. M. McCoy for the invaluable help for learning the integrable chiral Potts model. Through my collaboration with him, we found the mathematical structure involved in this subject revealed a surprising similarity from the physical and mathematical considerations. This strongly indicates the promising role of complex geometry on exactly solvable models. It is in this belief that this note began with. I also wish to thank Prof. R. J. Baxter for his beautiful preprint [6], in which the relation between Boltzmann weights and hyperelliptic theta functions are found via the classical work of Sonya Kowalevski [14]. One of the purposes of this paper is to understand the qualitative feature of the identities of different quantities in [6] from the symmetries of "rapidity" curves. I am most grateful to Prof. F. Hirzebruch for his kind invitation and the hospitality of MaxPlanck-Institut für Mathematik where this work was completed.

\section{Parametrization of 4 Points in $\mathbb{P}^{1}$}

Let $E$ be a 1-torus (=1-dimension complex torus), $T$ be the subgroup of Aut $(E)$ consisting of all translations of $E$, i.e. the automorphisms $t_{a}: E \rightarrow E, x \leadsto x+a$, $x \in E$. $\mathrm{T}$ is isomorphic to $E$. Denote $T(2)=$ the 2-torsion subgroup of $T$.

Let $\theta: E \rightarrow E$ be an involution with 4 fixed points.

Lemma 1. $T(2)=\{t \in T \mid t \theta=\theta t\}$.

Proof. Write $E=\mathbb{C} / L$, and $\sigma:[z] \rightarrow[-z+c]$ for some lattice $L$, and $c \in \mathbb{C}$. Then the conclusion follows easily from the definition of $T$. q.e.d.

Denote $H=(T(2) \times\langle\theta\rangle) /\langle\theta\rangle$. Identifying $E /\langle\theta\rangle$ with $\mathbb{P}^{1}, H$ can be considered as a subgroup of Aut $\left(\mathbb{P}^{1}\right)$ through its action on $E /\langle\theta\rangle$. The branched locus $S$ of the double cover

$$
\Psi\left(=\Psi_{\theta}\right):\left.E \rightarrow E\right|_{\langle\theta\rangle}=\mathbb{P}^{1}
$$

forms a $H$-orbit with 4 elements. As any two such involutions $\theta$ of $E$ are conjugate by a translation, the subset $S$ of $\mathbb{P}^{1}$ is uniquely determined up to the action of $\operatorname{Aut}\left(\mathbb{P}^{1}\right)$. It is well-known that every 1-torus can be obtained as a double cover of $\mathbb{P}^{1}$ with 4 branched points, hence we have the one-one correspondence between the following sets:

$$
\text { \{isomorphic class of 1-torus }\} \leftrightarrow\left\{S\left|S \subset \mathbb{P}^{1},\right| S \mid=4\right\} / \operatorname{Aut}\left(\mathbb{P}^{1}\right) \text {. }
$$

Definition. For a subset $S$ of $\mathbb{P}^{1}$ consisting of 4 elements, a partition of $S$ with 2 elements in each of its member is called a $(2,2)$-partition of $S$. 
We are going to characterize the data of $S$ with a (2,2)-partition. First we notice that the above group $H$ is determined by the set $S$ via (i) of the following lemma.

Lemma 2. (i) For each subset $S$ of $\mathbb{P}^{1}$ with $|S|=4$, there exists an unique subgroup $H$ of Aut $\left(\mathbb{P}^{1}\right)$ such that $H \simeq(\mathbb{Z} / 2 \mathbb{Z})^{2}$ and $S=$ a $H$-orbit.

(ii) Let $H$ be a subgroup of Aut $\left(\mathbb{P}^{1}\right)$ with $H \simeq(\mathbb{Z} / 2 \mathbb{Z})^{2}$. Then $\left(\mathbb{P}^{1}\right)^{h}, h \in H-\{$ id. $\}$, are mutually disjoint.

Proof. (i) Let $H$ be a subgroup of Aut $\left(\mathbb{P}^{1}\right)$ satisfying the condition. The $\langle h\rangle$ orbits in $S$ forms a (2,2)-partition of $S$ for a given non-trivial element $h$ of $H$. Then $h$ is uniquely determined by its values on $S$. Since $S$ has exact three (2,2)-partitions, $H$ is unique. It remains to show that for any $(2,2)$-partition of $S$, there always exists an automorphism $h$ of $\mathbb{P}^{1}$ such that the $\langle h\rangle$-orbits in $S$ form the given $(2,2)$ partition. By conjugating some element in Aut $\left(\mathbb{P}^{1}\right)$, we may assume $S=\{0, \infty, 1, \alpha\}$ and the partition $=\{0, \infty\} \sqcup\{1, \alpha\}$. Then $h$ is defined by $z \rightarrow \alpha / z$, $z \in \mathbb{C}$.

(ii) If $H$ has a common fixed point $x$ in $\mathbb{P}^{1}$, the homomorphism which assigns each element of $H$ to its differential at $x$ is an injective map. Since the linear transformation group of the tangent at $x$ is isomorphic to $\mathbb{C}^{*}, H$ is cyclic. This contradicts the assumption of the structure of $H$. q.e.d.

Proposition 1. $\{$ isomorphic class of $(E, t) \mid E$ : 1-torus, $t \in T(2)-\{\mathrm{id}\}$.$\} is in one-one$ correspondence with each of the following sets:

(i) $\left\{(S\right.$ with a (2,2)-partition $\left.)\left|S \subset \mathbb{P}^{1},\right| S \mid=4\right\} /$ Aut $\left(\mathbb{P}^{1}\right)$.

(ii) $\left\{\pi: \mathbb{P}^{1} \rightarrow \mathbb{P}^{1}\right.$, a degree 2 morphism with critical value not equal to $\left.0, \infty\right\} / \sim$, here $\pi_{1} \sim \pi_{2}$ iff for some $f, f^{\prime} \in$ Aut $\left(\mathbb{P}^{1}\right)$ with $f(\{0, \infty\})=\{0, \infty\}$,

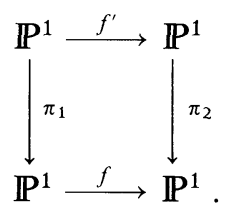

(iii) $\left\{(\varrho, p) \mid p \in \mathbb{P}^{1}-\{\infty\}\right.$ ' $\varrho$ : a degree 2 endomorphism of $\mathbb{P}^{1}$ with critical value not equal to $p, \infty\} / \approx$, here $\varrho_{1} \approx \varrho_{2}$ iff for some $f, g \in \operatorname{Aut}\left(\mathbb{P}^{1}\right)$ with $g(p)=p$, $g(\infty)=\infty$,

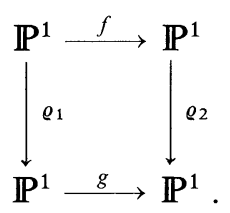

Proof. For $S \subset \mathbb{P}^{1}$ with $|S|=4$, let $E$ be the corresponding 1-torus of (2). The group $H$ in Lemma 2 (i) corresponding to the set $S$ can be identified with $(T(2) \times\langle\sigma\rangle) /\langle\sigma\rangle$. For $t$ in $T(2)-\{$ id. $\}$, let $h$ be the coset of $t$ in $H$. Then the $\langle h\rangle-$ orbits in $S$ forms a $(2,2)$-partition of $S$. In this way we define a map from \{isomorphism class of $(E, t) \mid t \in T(2)-\{$ id. $\}\}$ to (i), which is bijective by Lemma 2 .

For the above automorphism $h$ of $\mathbb{P}^{1}$, the degree 2 morphism $\pi: \mathbb{P}^{1} \rightarrow \mathbb{P}^{1} /\langle h\rangle=\mathbb{P}^{1}$ has the critical points disjoint with $S$. By a suitable coordinate of $\mathbb{P}^{1}$, we may assume that $S=\pi^{-1}(\{0, \infty\})$. In this way, we have the one-one correspondence between (i) and (ii). 
The projection

$$
\varrho: \mathbb{P}^{1} /\langle h\rangle \rightarrow \mathbb{P}^{1} / H,
$$

can be considered as a degree 2 endomorphism of $\mathbb{P}^{1}$. The $H$-orbit $S$ corresponds to a point of $\mathbb{P}^{1} / H=\mathbb{P}^{1}$, which equals to $\infty$ by a suitable coordinate of $\mathbb{P}^{1} / H$. The fixed point set of $h$ corresponds to another $H$-orbit $p$. Then $p$ is not equal to $\infty$ and the critical values of $\varrho$ can not be $p$ or $\infty$. This gives the correspondence between (ii) and (iii). q.e.d.

Then by (2), the following correspondence can easily be obtained.

Proposition 2. \{isomorphic class of 1-torus\} is in one-one correspondence with each of the following sets:

(i) $\left\{S\left|S \subset \mathbb{P}^{1},\right| S \mid=4\right\} /$ Aut $\left(\mathbb{P}^{1}\right)$.

(ii) $\left\{\pi: \mathbb{P}^{1} \rightarrow \mathbb{P}^{1}\right.$ degree 2 morphism with critical value not equal to $\left.0, \infty\right\} / \simeq$, here $\pi_{1} \simeq \pi_{2}$ iff for some $f^{\prime}$ in Aut $\left(\mathbb{P}^{1}\right), f^{\prime}$ sends $\pi_{1}^{-1}\{0, \infty\}$ to $\pi_{2}^{-1}\{0, \infty\}$.

(iii) $\left\{(\varrho, p) \mid p \in \mathbb{P}^{1}-\{\infty\}, \varrho:\right.$ a degree 2 morphism of $\mathbb{P}^{1}$ onto itself with critical value not equal to $p, \infty\} / \approx$, here $\left(\varrho_{1}, p_{1}\right) \approx\left(\varrho_{2}, p_{2}\right)$ iff there exists some $g$ in Aut $\left(\mathbb{P}^{1}\right)$ such that $g(\infty)=\infty$, and $g\left(\left\{p_{1}\right.\right.$, critical values of $\left.\left.\varrho_{1}\right\}\right)=\left\{p_{2}\right.$, critical values of $\left.\varrho_{2}\right\}$.

We are going to parametrize the data in Proposition 1 and 2. The following easy lemma is useful for our derivation, and its proof is omitted.

Lemma 3. For 4 distinct elements $x, y, z, w$ in $\mathbb{P}^{1}$, there exists an automorphism $g$ of $\mathbb{P}^{1}$ with $g(x)=0, g(y)=\infty, g(z)=\alpha, g(w)=\alpha^{-1}$ for some $\alpha$ in $\mathbb{C}-\{0, \pm 1\}$. And $\alpha$ is unique up to sign.

Define

$$
\pi_{\alpha}: \mathbb{P}^{1} \rightarrow \mathbb{P}^{1}, \quad \lambda \leadsto \pi_{\alpha}(\lambda)=\frac{(1-\alpha \lambda)\left(1-\alpha \lambda^{-1}\right)}{1-\alpha^{2}},
$$

here $\alpha$ is a constant in $\mathbb{C}-\{0, \pm 1\}$

$$
\begin{gathered}
\varrho_{0}: \mathbb{P}^{1} \rightarrow \mathbb{P}^{1}, \quad \zeta \leadsto \frac{\zeta+\zeta^{-1}}{2}, \\
\mathscr{A}=\mathbb{C}-\{0, \pm 1\} / \equiv, \quad \text { here } \alpha \equiv \beta \text { iff } \alpha^{2}=\beta^{ \pm 2} .
\end{gathered}
$$

Proposition 3. $\mathscr{A}$ is in one-one correspondence with the data in Proposition 1. In fact, for an element $[\alpha]$ of $\mathscr{A}$ determined by a complex number $\alpha$, the elements of (i), (ii), (iii) in Proposition 1 corresponding to $[\alpha]$ are as follows:

(i) the class of $\left\{0, \infty, \alpha, \alpha^{-1}\right\}$ with the partition $\{0, \infty\} \sqcup\left\{\alpha, \alpha^{-1}\right\}$.

(ii) the class of $\pi_{\alpha}$.

(iii) the class of $\left(\varrho_{0}, \frac{1+\alpha^{2}}{1-\alpha^{2}}\right)$.

Proof. By Lemma 3, every element in (i) of Proposition 1 is represented by $\left\{0, \infty, \alpha, \alpha^{-1}\right\}$ with the partition $\{0, \infty\} \sqcup\left\{\alpha, \alpha^{-1}\right\}$ for some $\alpha \in \mathbb{C}-\{0, \pm 1\}$. This corresponds to an element of (ii) in Proposition 1, which is represented by $\pi: \mathbb{P}^{1} \rightarrow \mathbb{P}^{1}$. Choose the coordinate $\lambda$ of $\mathbb{C}\left(\subset \mathbb{P}^{1}\right)$ such that $\pi^{-1}(\infty)=\{0, \infty\}$, $\pi^{-1}(0)=\left\{\alpha, \alpha^{-1}\right\}$. Then $\pi$ is equivalent to $\pi_{\alpha}$. Since the critical values of $\pi_{\alpha}$ are $\frac{1-\alpha}{1+\alpha}, \frac{1+\alpha}{1-\alpha}$, the element in Proposition 1 (iii) is represented by $(\varrho, p)$ with 
$\varrho^{-1}(\infty)=\{0, \infty\}, \varrho^{-1}(p)=\left\{\frac{1-\alpha}{1+\alpha}, \frac{1+\alpha}{1-\alpha}\right\}$. So $\varrho$ is equivalent to $\left(\varrho_{0}, \frac{1+\alpha^{2}}{1-\alpha^{2}}\right)$. It remains to show that if $\left(\varrho_{0}, \frac{1+\alpha^{2}}{1-\alpha^{2}}\right)$ is equivalent to $\left(\varrho_{0}, \frac{1+\beta^{2}}{1-\beta^{2}}\right)$, then $[\alpha]=[\beta]$, i.e. $\beta^{2}=\alpha^{ \pm 2}$. Let $f, g$ be the elements of Aut $\left(\mathbb{P}^{1}\right)$ with $f \varrho_{0}=\varrho_{0} g$, and $g(\infty)=\infty$, $g\left(\frac{1+\alpha^{2}}{1-\alpha^{2}}\right)=\frac{1+\beta^{2}}{1-\beta^{2}}$. Then $f\{0, \infty\}=\{0, \infty\}$ and $f(\{1,-1\})=\{1,-1\}$, hence $f$ is defined by $f(\zeta)= \pm \zeta^{ \pm 1}$. Therefore $g$ is defined by $g(\zeta)= \pm \zeta$, which implies $\beta^{2}=\alpha^{ \pm 2}$. q.e.d.

Lemma 4. For a subset $S$ of $\mathbb{P}^{1}$ with 4 elements, let $H$ be the subgroup of Aut $\left(\mathbb{P}^{1}\right)$ associated to $S$ in Lemma 2 , and $\Phi: \mathbb{P}^{1} \rightarrow \mathbb{P}^{1} / H$ be the natural projection. Then the following data are in one-one correspondence:

$$
\{(2,2)-\text { partition of } S\} \leftrightarrow H-\{\text { id. }\} \leftrightarrow\{\text { critical value of } \Phi\}
$$

$\Delta \quad \leadsto h \leftrightarrow h \leftrightarrow c$

with the relation $\Delta=$ the $\langle h\rangle$-orbits decomposition of $S,\left(\mathbb{P}^{1}\right)^{h}=\Phi^{-1}(c)$.

Proof. For a critical value $c$ of $\Phi, \Phi^{-1}(c)$ is the fixed point set of a non-trival element of $H$, and vice versa. Then the conclusion follows immediately from Lemma 2. q.e.d.

With the same convention as in $[1,6,10]$, we shall always denote $k$ the solution of the equation

$$
k^{2}+k^{\prime 2}=1
$$

for a given $k^{\prime} \in \mathbb{C}-\{0, \pm 1\}$, and $\lambda\left(\right.$ or $\left.\lambda_{j}\right)$ the coordinate of the domain of $\pi_{\alpha}$.

Proposition 4. (i) For $\left[k^{\prime}\right] \in \mathscr{A}$, the elements of $\mathscr{A}$ having the same image as $\left[k^{\prime}\right]$ in Proposition 2 are: $\left[k^{\prime}\right],[k],\left[\frac{i k^{\prime}}{k}\right]$.

(ii) For $S=\left\{0, \infty, k^{\prime}, k^{\prime-1}\right\}$, the elements in (i) of Proposition 1 corresponding to the above three elements of $\mathscr{A}$ are as follows:

$\left[k^{\prime}\right] \leadsto S$ with the partition $\{0, \infty\} \sqcup\left\{k^{\prime}, k^{\prime-1}\right\}$,

$$
\begin{gathered}
{\left[\frac{i k^{\prime}}{k}\right] \leadsto S \text { with the partition }\left\{0, k^{\prime-1}\right\} \sqcup\left\{\infty, k^{\prime}\right\},} \\
{[k] \leftrightarrow S \text { with the partition }\left\{0, k^{\prime}\right\} \sqcup\left\{\infty, k^{\prime-1}\right\} .}
\end{gathered}
$$

(iii) Let $\zeta, \zeta_{1}, \zeta_{2}$ be the coordinates of the domains of $\varrho_{0}$ in (iii) of Proposition 3 such that:

$$
\left[k^{\prime}\right] \leftrightarrow\left(\varrho_{0}, \frac{1+k^{\prime 2}}{1-k^{\prime 2}}\right) \text { with the coordinate } \zeta,
$$$$
\left[\frac{i k^{\prime}}{k}\right] \leadsto\left(\varrho_{0}, 1-2 k^{\prime 2}\right) \text { with the coordinate } \zeta_{1} \text {, }
$$

$[k] \leftrightarrow\left(\varrho_{0}, \frac{1+k^{2}}{1-k^{2}}\right)$ with the coordinate $\zeta_{2}$. 
Then $\zeta, \zeta_{1}, \zeta_{2}$ are related by

$$
\begin{gathered}
\zeta_{1}+\zeta_{1}+2=k^{2}\left(\zeta+\zeta^{-1}+2\right), \\
\zeta_{2}+\zeta_{2}^{-1}-2=-\frac{k^{2}}{k^{\prime 2}}\left(\zeta+\zeta^{-1}-2\right) .
\end{gathered}
$$

Proof. In this proof, we shall always denote $k^{\prime}$ an element in $\mathbb{C}-\{0, \pm 1\}$, $S=\left\{0, \infty, k^{\prime}, k^{\prime-1}\right\}, H=$ the order 4 subgroup of Aut $\left(\mathbb{P}^{1}\right)$ associated to $S$ in (i) of Lemma $2, \zeta, \zeta_{1}, \zeta_{2}=$ the three coordinates for $\mathbb{P}^{1}$ in the above (iii) of this proposition. Let $h, h_{1}, h_{2}$ be the non-trivial elements of $H$ such that the correspondence in Lemma 4 are as follows:

$$
\begin{gathered}
h \leftrightarrow\{0, \infty\} \sqcup\left\{k^{\prime}, k^{\prime-1}\right\}, \\
h_{1} \leadsto\left\{0, k^{\prime-1}\right\} \sqcup\left\{\infty, k^{\prime}\right\}, \\
h_{2} \leadsto\left\{0, k^{\prime}\right\} \sqcup\left\{\infty, k^{\prime-1}\right\} .
\end{gathered}
$$

Identify the projection $\mathbb{P}^{1} \rightarrow \mathbb{P}^{1} /\langle h\rangle$ with $\pi_{k^{\prime}}, \mathbb{P}^{1} /\langle h\rangle \rightarrow \mathbb{P}^{1} / H$ with $\varrho_{0}$, and $\Phi$ : $\mathbb{P}^{1} \rightarrow \mathbb{P}^{1} / H$ with $\varrho_{0} \pi_{k^{\prime}}$. Then $\{$ critical values of $\Phi\}=\left\{ \pm 1, \frac{1+k^{\prime 2}}{1-k^{\prime 2}}\right\}$, and $h, h_{1}, h_{2}$
are defined by

$$
h(\lambda)=\lambda^{-1}, \quad h_{1}(\lambda)=\frac{1-k^{\prime} \lambda}{k^{\prime}-\lambda}, \quad h_{2}(\lambda)=\frac{k^{\prime}-\lambda}{1-k^{\prime} \lambda} .
$$

The correspondence between the critical values of $\Phi$ and the elements of $H-\{\mathrm{id}$. in Lemma 4 is described as follows:

$$
h \leftrightarrow \frac{1+k^{\prime 2}}{1-k^{\prime 2}}, \quad h_{1} \leadsto 1, \quad h_{2} \leadsto-1 .
$$

Let $[\alpha]$ be an element of $\mathscr{A}$ having the same image as $\left[k^{\prime}\right]$ in Proposition 2, and assume $[\alpha] \neq\left[k^{\prime}\right]$. Then $\left(\varrho_{0}, \frac{1+k^{\prime 2}}{1-k^{\prime 2}}\right)$ and $\left(\varrho_{0}, \frac{1+\alpha^{2}}{1-\alpha^{2}}\right)$ are equivalent under the relation $\approx$ in (iii) of Proposition 2, i.e. for some $g$ in $\operatorname{Aut}\left(\mathbb{P}^{1}\right), g(\infty)=(\infty)$, $g\left(\left\{\frac{1+k^{\prime 2}}{1-k^{\prime 2}}, \pm 1\right\}\right)=\left\{\frac{1+\alpha^{2}}{1-\alpha^{2}}, \pm 1\right\}$. By (iii) of Proposition 1, we may assume $g\left(\frac{1+k^{\prime 2}}{1-k^{\prime 2}}\right)= \pm 1$. Then the $g$ is uniquely determined by the value of 1 . When $\left(g\left(\frac{1+k^{\prime 2}}{1-k^{\prime 2}}\right), g(1)\right)=\left(1, \frac{1+\alpha^{2}}{1-\alpha^{2}}\right)$ or $\left(-1, \frac{1+\alpha^{2}}{1-\alpha^{2}}\right), g(-1)=-1$ or 1 respectively. It follows $g(\eta)= \pm\left(k^{2} \eta-k^{\prime 2}\right)$ which implies $\alpha^{2}=\frac{-k^{\prime 2}}{k^{2}}$ or $\frac{-k^{2}}{k^{\prime 2}}$, hence $[\alpha]=\left[\frac{i k^{\prime}}{k}\right]$. In this cases, the $(2,2)$-partition of $S$ is given by the $\left\langle h_{1}\right\rangle$-orbits. When $\alpha^{2}=\frac{-k^{2}}{k^{2}}$, we have

$$
k^{2}\left(\frac{\zeta+\zeta^{-1}}{2}\right)-k^{\prime 2}=\frac{\zeta_{1}+\zeta_{1}^{-1}}{2}
$$

hence

$$
\zeta_{1}+\zeta_{1}^{-1}+2=k^{2}\left(\zeta+\zeta^{-1}+2\right) .
$$


When $\left(g\left(\frac{1+k^{\prime 2}}{1-k^{\prime 2}}\right), g(1)\right)=(1,-1)$ or $(-1,1)$, the (2,2)-partition of $S$ is given by the $\left\langle h_{2}\right\rangle$-orbits and $g(\eta)= \pm\left(k^{2} \eta-1\right) / k^{\prime 2}$. This implies $\alpha^{2}=k^{-2}$ or $k^{2}$, hence $[\alpha]=[k]$. When $\alpha^{2}=k^{2}$, we have

$$
\frac{1}{k^{\prime 2}}\left[-k^{2}\left(\frac{\zeta+\zeta^{-1}}{2}\right)+1\right]=\frac{\zeta_{2}+\zeta_{2}^{-1}}{2},
$$

hence

$$
\zeta_{2}+\zeta_{2}^{-1}-2=-\frac{k^{2}}{k^{\prime 2}}\left(\zeta+\zeta^{-1}-2\right)
$$

This completes the proof of this proposition. q.e.d.

Remark.

(i) By Proposition 1 (ii), there is an automorphism $f^{\prime}$ of $\mathbb{P}^{1}$ which sends $\left\{0, k^{\prime-1}\right\} \sqcup\left\{\infty, k^{\prime}\right\}$ to $\{0, \infty\} \sqcup\left\{-i \frac{k}{k^{\prime}}, i \frac{k^{\prime}}{k}\right\}$. In fact, $f^{\prime}$ is defined by

$$
f^{\prime}(\lambda)=\frac{i k \lambda}{1-k^{\prime} \lambda} \text {. }
$$

And the $f^{\prime}$ sending $\left\{0, k^{\prime}\right\} \sqcup\left\{\infty, k^{\prime-1}\right\}$ to $\{0, \infty\} \sqcup\left\{k, k^{-1}\right\}$ is defined by $f^{\prime}(\lambda)=\frac{k \lambda}{\lambda-k^{\prime}}$.

(ii) By introducing the variables $u, \phi, \bar{\phi}$

$$
-\zeta=e^{2 i u}, \quad-\zeta_{1}=e^{2 i \phi}, \quad \zeta_{2}=e^{2 i \bar{\phi}},
$$

the relations of (iii) in the above proposition are equivalent to

$$
\sin \phi= \pm k \sin u, \quad k^{\prime} \sin \bar{\phi}= \pm i k \cos u,
$$

which are the relations of the parameters for " $p$-variables" in $[1,5,6,10]$. The variables $e^{i u}, e^{i \phi}, e^{i \bar{\phi}}(\operatorname{as} \operatorname{Im}(u), \operatorname{Im}(\phi), \operatorname{Im}(\phi), \operatorname{Im}(\bar{\phi}) \rightarrow \infty)$ can be considered as the local coordinates of the 2-torsion points of the elliptic curve corresponding to $[k]$ in Proposition 2.

\section{Chiral Potts $N$-State Curves}

The algebraic curve where the "rapidity" variables of chiral Potts $N$-state model $(N \geqq 3)$ lie is defined as follows:

Definition. The chiral Potts $N$-state curve $(N \geqq 3)$ is a genus $(N-1)$ hyperelliptic curve $W$ with an order $N$ automorphism having exactly 4 fixed points. And we shall call $W$ a CP $N$-curve.

The order $N$ automorphism of $W$ in the above definition shall always be denoted by $\theta\left(=\theta_{W}\right)$, and the hyperelliptic involution be denoted by $\sigma\left(=\sigma_{W}\right)$. By the uniqueness of the hyperelliptic involution, $\theta$ commutes with $\sigma$. First we note that the following properties hold for CP $N$-curves.

(I) $\langle\theta\rangle$ acts freely on $W-W^{\theta}$, and $W /\langle\theta\rangle \simeq \mathbb{P}^{1}$.

Let $g_{0}$ be the genus of $W \mid\langle\theta\rangle$. By the Hurwitz theorem for the projection from $W$ to $W \mid\langle\theta\rangle, 4-2 N=N\left(2-2 g_{0}\right)-4(N-1)-\star$, here $\star=$ the sum of 
ramification contribution of $x \in W-W^{\theta}$. Hence $0=2 N g_{0}+\star$, and $g_{0}=\star=0$. Then the conclusion follows immediately.

(II) $W^{\theta}$ is disjoint with $W^{\sigma}$.

By the commutativity of $\theta$ and $\sigma, W^{\theta}$ is stable under $\sigma$. Hence $\left|W^{\theta} \cap W^{\sigma}\right|=2$ or 4 when it is non-zero. As $\langle\theta\rangle$ acts freely on $W^{\sigma}-W^{\theta}$, the order of $W^{\sigma}-W^{\theta}$, $(=2 N-2$ or $2 N-4)$, is divisible by $N$. By $N \geqq 3$, this is only possible for $N=4$, and $\left|W^{0} \cap W^{\sigma}\right|=\left|W^{0}\right|=4$. By Hurwitz's Theorem, the sum of all the ramification contribution of elements in $W$ for the projection $W \rightarrow W /\langle\theta, \sigma\rangle\left(=\mathbb{P}^{1}\right)$ equal to 20 , which is greater than the contribution from the 4 elements in $W^{\theta}$. This gives a contradiction because the ramification contribution of each element in $W^{\theta}$ is 7 . Therefore $W^{\theta} \cap W^{\sigma}=\emptyset$.

Now we are going to describe the algebraic form of CP $N$-curves. Let $\Psi, \psi, \Pi, \pi$ be the morphisms defined by the following commutative diagram:

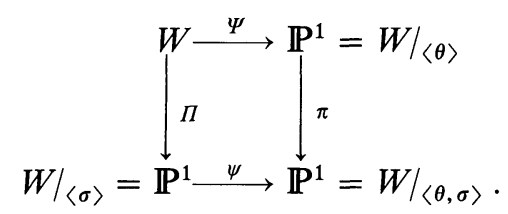

Let $S$ be the branched locus of $\Psi$, and $h$ be the automorphism of $\mathbb{P}^{1}(=W /\langle\theta\rangle)$ induced by $\sigma$. By the above (II), the orbits of the automorphism of $W /\langle\theta\rangle$ induced by $\sigma$ form a (2,2)-partition of $S$. By Proposition 3 , for some coordinate $\lambda$ of $\mathbb{P}^{1}$ and some $k^{\prime} \in \mathbb{C}-\{0, \pm 1\}, S=\{0, \infty\} \sqcup\left\{k^{\prime}, k^{\prime-1}\right\}$, and $\pi=\pi_{k^{\prime}}$. Choose the coordinate $t$ of $\mathbb{P}^{1}(=W /\langle\sigma\rangle)$ such that $\psi$ is defined by $t \leadsto t^{N}$. Then $W$ is isomorphic to

$$
W_{N, k^{\prime}}: t^{N}=\frac{\left(1-k^{\prime} \lambda\right)\left(1-k^{\prime} \lambda^{-1}\right)}{k^{2}} .
$$

In terms of the coordinate $(t, \lambda), \theta$ and $\sigma$ are defined by

$$
\begin{aligned}
& \theta:(t, \lambda) \leadsto(\omega t, \lambda), \\
& \sigma:(t, \lambda) \leadsto\left(t, \lambda^{-1}\right), \quad \text { here } \omega=e^{2 \pi i / N} .
\end{aligned}
$$

Since the branched locus of $\Pi$ is equal to

$$
\left\{b \mid b^{N}=\frac{1-k^{\prime}}{1+k^{\prime}} \quad \text { or } \quad \frac{1+k^{\prime}}{1-k^{\prime}}\right\} \text {. }
$$

$W_{N, k^{\prime}}$ is also birationally equivalent to the curve in $\mathbb{C}^{2}$ defined by the equation:

$$
w^{2}=\left(t^{N}-\frac{1-k^{\prime}}{1+k^{\prime}}\right)\left(t^{N}-\frac{1+k^{\prime}}{1-k^{\prime}}\right), \quad(t, w) \in \mathbb{C}^{2}
$$

The above coordinates $\lambda, w$ are related by

$$
\begin{gathered}
w=\frac{k^{\prime}}{k^{2}}\left(\lambda-\frac{1}{\lambda}\right), \\
\lambda=\frac{1}{2 k^{\prime}}\left\{k^{2}\left(w-t^{N}\right)+k^{\prime 2}+1\right\} .
\end{gathered}
$$


For the rest of this note, $(t, \lambda)$ and $(t, w)$ shall always denote the above coordinates for the curve $W_{N, k^{\prime}}$. In terms of the coordinate $(t, w), \theta$ and $\sigma$ are defined by

$$
\begin{aligned}
& \theta:(t, w) \leadsto(\omega t, w), \\
& \sigma:(t, w) \leadsto(t,-w) .
\end{aligned}
$$

Let $l\left(=l_{W_{N, k^{\prime}}}\right)$ be the order 2 automorphism of $W_{N, k^{\prime}}$ defined by

$$
l: W_{N, k^{\prime}} \rightarrow W_{N, k^{\prime}}, \quad(t, w) \leadsto\left(\frac{1}{t}, \frac{w}{t^{N}}\right),
$$

which is the same as

$$
l:(t, \lambda) \leadsto\left(\frac{1}{t}, \frac{1-k^{\prime} \lambda}{k^{\prime}-\lambda}\right)
$$

by the equality

$$
\frac{1}{2}\left(\frac{\lambda-\lambda^{-1}}{t^{N}}-\frac{k^{2}}{k^{\prime} t^{N}}+k^{\prime}+\frac{1}{k^{\prime}}\right)=\frac{1-k^{\prime} \lambda}{k^{\prime}-\lambda}
$$

Then $\theta \imath=\imath \theta^{-1}$, and $\langle\theta, \imath\rangle \simeq$ the subgroup $\overline{D_{N}}$ of Aut $\left(\mathbb{P}^{1}\right)$ with the binary dihedral group $D_{N}$ as its double cover. Now we can described all the symmetries of a CP $N$-curve.

Proposition 5. For a CP $N$-curve $W$, Aut $(W)=\langle\sigma, \theta, \imath\rangle \simeq(\mathbb{Z} / 2 \mathbb{Z}) \times \overline{D_{N}}$, and $\langle\theta\rangle$ is the unique order $N$ cyclic subgroup of Aut $(W)$ generated by an automorphism with non-empty fixed point set.

Proof. Since $\sigma$ commutes with any automorphism of $W$, the group Aut $(W) /\langle\sigma\rangle$ acts on $\mathbb{P}^{1}(=W /\langle\sigma\rangle)$ and preserves the branched locus (6). It is not hard to see $\langle\theta, \imath\rangle \simeq \operatorname{Aut}(W)\langle\sigma\rangle \simeq \overline{D_{N}}$, hence $\operatorname{Aut}(W) \simeq(\mathbb{Z} / 2 \mathbb{Z}) \times \overline{D_{N}}$. For an order $N$ automorphism $\phi$ of $W$ with non-empty fixed part $W^{\phi}$, write $\phi=\sigma^{m} l^{n} \theta^{j}$, for some integers $0 \leqq m, n \leqq 1,0 \leqq j \leqq(N-1)$. We need to show $m=n=0$. Since $l \cdot \theta^{j}$ is of order $2, n$ has to be 0 . If $\phi=\sigma \cdot \theta^{j}, j$ is greater than 0 . The image of the fixed point set of $\phi$ under the projection $\Pi$ in (3) is contained in the fixed part of the automorphism of $W\left\langle\langle\sigma\rangle\right.$ induced by $\theta^{j}$. Hence $W^{\phi}$ is contained in $W^{\theta}$, then also in $W^{\sigma}$. But the fixed parts of $\theta$ and $\sigma$ are disjoint. This contradicts the assumption of the non-emptyness of the fixed point set of $\phi$. Therefore $j=0$, and $\langle\phi\rangle=\langle\theta\rangle$. q.e.d.

Corollary. The morphism $\Psi: W \rightarrow \mathbb{P}^{1}$ is the unique (up to isomorphism) order $N$ cyclic covering of $W$ over $\mathbb{P}^{1}$ with exactly 4 distinct critical values.

Proof. Let $\phi$ be an order $N$ automorphism of $W$ such that the projection $W \rightarrow W \mid\langle\phi\rangle=\mathbb{P}^{1}$ has exactly 4 critical values. By Hurwitz's Theorem, $\left|W^{\phi}\right|=4$. Hence $\langle\phi\rangle=\langle\theta\rangle$ by the above proposition, and the conclusion follows immediately. q.e.d.

Corollary. The curves $W_{N, \alpha}$ and $W_{N, \beta}$ are isomorphic as Riemann surfaces if and only if $[\alpha]=[\beta]$ in $\mathscr{A}$. 
Proof. Let $\phi$ be the biregular isomorphism from $W_{N, \alpha}$ to $W_{N, \beta}$. Then

$$
\begin{aligned}
& \phi^{-1} \sigma_{W_{N, \beta}} \cdot \phi=\sigma_{W_{N, \alpha}} \text { (by the uniqueness of hyperelliptic involution), } \\
& \phi^{-1} \theta_{W_{N, \beta}} \cdot \phi=\theta_{W_{N, \alpha}} \text { (by the above Corollary). }
\end{aligned}
$$

Hence the diagrams (3) for the curves $W_{N, \alpha}$ and $W_{N, \beta}$ are equivalent through the morphisms induced by $\phi$. This is equivalent to $[\alpha]=[\beta]$ in $\mathscr{A}$ because the $(2,2)$ partition of the branched locus of $\Psi$ for $W_{N, \alpha}$ and $W_{N, \beta}$ are equivalent in the relation of Proposition 1 (i). q.e.d.

\section{Three Related Chiral Potts $N$-Curves}

We continue to study the $\mathrm{CP} N$-curves. By the Corollary of Proposition 5, the CP $N$-curves are parametrized by $\mathscr{A}$. For a given $k^{\prime} \in \mathbb{C}-\{0, \pm 1\}$, the elements $\left[k^{\prime}\right]$, $\left[\frac{i k^{\prime}}{k}\right],[k]$ of $\mathscr{A}$ are related in the sense that they determine the same 1-torus in Proposition 2 by the result of Proposition 4 . For a given $k^{\prime} \in \mathbb{C}-\{0, \pm 1\}$, we are going to study the relation between the three CP $N$-curves $W_{N, k^{\prime}}, W_{N, i k^{\prime} / k}, W_{N, k}$. The curve $W_{N, k^{\prime}}$ is defined by the coordinate $(t, \lambda)$ of (4). By (ii) of Proposition 3 , we have

$$
\begin{aligned}
& W_{N, i k^{\prime} \mid k} \simeq W_{r}: \\
& t_{r}^{N}=\frac{1-k^{\prime} \lambda}{1-k^{\prime} \lambda^{-1}}, \quad\left(t_{r}, \lambda\right) \in \mathbb{C}^{2},
\end{aligned}
$$

and the automorphisms $\sigma, l$ for $W_{r}$ are defined by

$$
\begin{aligned}
& \sigma_{W_{r}}:\left(t_{r}, \lambda\right) \leadsto\left(t_{r}, \frac{1-k^{\prime} \lambda}{k^{\prime}-\lambda}\right), \\
& l_{W_{r}}:\left(t_{r}, \lambda\right) \leadsto\left(\frac{1}{t_{r}}, \frac{1}{\lambda}\right) .
\end{aligned}
$$

Similarly, $W_{N, k} \simeq W_{l}$ :

$$
t_{l}^{N}=\frac{k^{\prime}-\lambda^{-1}}{k^{\prime}-\lambda}, \quad\left(t_{l}, \lambda\right) \in \mathbb{C}^{2},
$$

with the automorphisms

$$
\begin{aligned}
& \sigma_{W_{l}}:\left(t_{l}, \lambda\right) \leadsto\left(t_{l}, \frac{k^{\prime}-\lambda}{1-k^{\prime} \lambda}\right), \\
& \iota_{W_{l}}:\left(t_{l}, \lambda\right) \leadsto\left(\frac{1}{t_{l}}, \frac{1}{\lambda}\right) .
\end{aligned}
$$

In this section, we shall write $W=W_{N, k^{\prime}}$. The morphisms $\Psi, \Psi_{r}, \Psi_{l}$ from $W, W_{r}$, $W_{l}$ to $\mathbb{P}^{1}$ in (3) are now defined by $\Psi(t, \lambda)=\lambda, \Psi_{r}\left(t_{r}, \lambda\right)=\lambda, \Psi_{l}\left(t_{l}, \lambda\right)=\lambda$. 
Denote the fiber product $W \times_{\mathbb{P}^{1}} W_{r}$ of $\Psi$ and $\Psi_{r}$ by $\mathscr{W}_{r}$ :

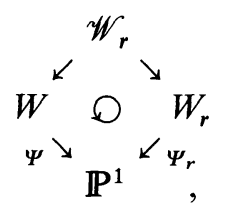

and $\mathscr{W}_{r}$ is defined by

$$
\begin{aligned}
& t^{N}=\frac{\left(1-k^{\prime} \lambda\right)\left(1-k^{\prime} \lambda^{-1}\right)}{k^{2}} \\
& t_{r}^{N}=\frac{1-k^{\prime} \lambda}{1-k^{\prime} \lambda^{-1}}, \quad \text { for }\left(t, t_{r}, \lambda\right) \in \mathbb{C}^{3} .
\end{aligned}
$$

The automorphisms $\theta_{W}, \theta_{W_{r}}$ of $W, W_{r}$ induce the automorphisms $\Theta, \Theta_{r}$ of $\mathscr{W}_{r}$ which are described by

$$
\begin{gathered}
\Theta:\left(t, t_{r}, \lambda\right) \leadsto\left(\omega t, t_{r}, \lambda\right), \\
\Theta_{r}:\left(t, t_{r}, \lambda\right) \rightsquigarrow\left(t, \omega t_{r}, \lambda\right) .
\end{gathered}
$$

Then the group $\left\langle\Theta, \Theta_{r}\right\rangle$ isomorphic to $(\mathbb{Z} / N \mathbb{Z})^{2}$, and acts freely on $\mathscr{W}_{r}$ outside the 4 critical points of the projection from $\mathscr{W}_{r}$ to the $\lambda$-plane, which has the branched locus $\left\{0, \infty, k^{\prime}, k^{\prime-1}\right\}$. Consider the quotients:

$$
V_{1}=\left.\mathscr{W}_{r}\right|_{\left\langle\theta \Theta_{r}\right\rangle}, \quad V_{2}=\left.\mathscr{W}_{r}\right|_{\left\langle\Theta \Theta_{r}^{-1}\right\rangle} .
$$

Then $V_{1}, V_{2}$ are $N$-sheet cover of the $\lambda$-plane $\mathbb{P}^{1}$ branched at $\left\{k^{\prime}, 0\right\}$ and $\left\{\frac{1}{k^{\prime}}, \infty\right\}$ respectively with the following coordinates:

$$
\begin{array}{ll}
V_{1}:\left(\frac{t}{t_{r}}\right)^{N}=\frac{\left(1-k^{\prime} \lambda^{-1}\right)^{2}}{k^{2}}, & \left(\frac{t}{t_{r}}, \lambda\right) \in \mathbb{C}^{2} ; \\
V_{2}:\left(t t_{r}\right)^{N}=\frac{\left(1-k^{\prime} \lambda\right)^{2}}{k^{2}}, & \left(t t_{r}, \lambda\right) \in \mathbb{C}^{2} .
\end{array}
$$

Consider the degree 2 cover of the $\left(\frac{t}{t_{r}}\right)$-plane,

$$
\mathbb{P}^{1} \rightarrow \mathbb{P}^{1}, \quad x \rightsquigarrow\left(\frac{t}{t_{r}}\right)=x^{2} .
$$

The fiber product of $x$-plane with $V_{1}$ over the $\left(\frac{t}{t_{r}}\right)$-plane is reducible with two rational curves as its irreducible components. They are described by

$$
x^{N}= \pm \frac{1-k^{\prime} \lambda^{-1}}{k}, \quad(x, \lambda) \in \mathbb{C}^{2} .
$$

Let $X$ be the component

$$
x^{N}=\frac{1-k^{\prime} \lambda^{-1}}{k}, \quad(x, \lambda) \in \mathbb{C}^{2} .
$$


$X$ is a $N$-fold cover of the $\lambda$-plane $\mathbb{P}^{1}$ branched at $\left\{k^{\prime}, 0\right\}$. With the same discussion for $V_{2}$, we have a $N$-fold cover $Y$ of the $\lambda$-plane $\mathbb{P}^{1}$ branched at $\left\{\frac{1}{k^{\prime}}, \infty\right\}$ which is
defined by

$$
y^{N}=\frac{1-k^{\prime} \lambda}{k}, \quad(y, \lambda) \in \mathbb{C}^{2} .
$$

There are the morphisms from the fiber product of $X$ and $Y$ over the $\lambda$-plane into $W, W_{r}$ with the following commutative diagram:

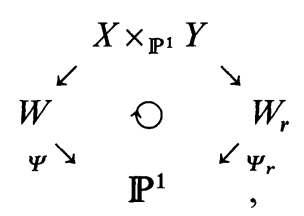

which are described by:

$$
(x y, \lambda)=(t, \lambda)^{\swarrow \iota_{\lambda}^{(x, y, \lambda)} \swarrow^{\left(t_{r}, \lambda\right)}=\left(\frac{y}{x}, \lambda\right)}
$$

Similarly by replacing the above $W_{r}$ by $W_{l}$, we have the rational curves $X^{\prime}, Y^{\prime}$ with the degree $N$ morphisms over the $\lambda$-plane:

$$
\begin{aligned}
X^{\prime} \rightarrow \mathbb{P}^{1}, \quad x^{\prime} \rightsquigarrow \lambda, \quad \text { with } \quad x^{\prime N}=\frac{\lambda-k^{\prime}}{k}, \quad\left(x^{\prime 2}=\frac{t}{t_{l}}\right), \\
Y^{\prime} \rightarrow \mathbb{P}^{1}, \quad y^{\prime} \rightsquigarrow \lambda, \quad \text { with } \quad y^{\prime N}=\frac{\lambda^{-1}-k^{\prime}}{k}, \quad\left(y^{\prime 2}=t t_{l}\right),
\end{aligned}
$$

and also the diagram:

$$
\begin{array}{lll}
W_{l} \searrow & \bigcirc & X^{\prime} \times_{\mathbb{P}^{1}} Y^{\prime} \\
\Psi_{l} \searrow & \mathbb{P}^{1} & \swarrow_{\Psi}
\end{array}
$$

defined by

$$
\left(\frac{y^{\prime}}{x^{\prime}}, \lambda\right)=\left(t_{l}, \lambda\right) \swarrow_{\searrow}^{\left(x^{\prime}, y^{\prime}, \lambda\right)} \stackrel{\swarrow}{\searrow}^{(t, \lambda)}=\left(x^{\prime} y^{\prime}, \lambda\right)
$$

The coordinates of $X, X^{\prime}$ and $Y, Y^{\prime}$ are related by:

$$
\left(\frac{x}{x^{\prime}}\right)^{N}=\lambda^{-1}, \quad\left(\frac{y}{y^{\prime}}\right)^{N}=\lambda .
$$


It follows that under the base change

$$
\mathbb{P}_{\mu}^{1} \rightarrow \mathbb{P}^{1,} \quad \mu \rightsquigarrow \lambda=\mu^{N},
$$

we have

$$
X \times_{\mathbb{P}^{1}} \mathbb{P}_{\mu}^{1} \simeq X^{\prime} \times_{\mathbb{P}^{1}} \mathbb{P}_{\mu}^{1}, \quad Y \times_{\mathbb{P}^{1}} \mathbb{P}_{\mu}^{1} \simeq Y^{\prime} \times_{\mathbb{P}^{1}} \mathbb{P}_{\mu}^{1} .
$$

In fact, these are the Fermat curves in $\mathbb{P}^{2}$ :

$$
\begin{array}{rlrl}
\mathfrak{X} & =X \times_{\mathbb{P}^{1}} \mathbb{P}_{\mu}^{1}, & & k a^{N}+k^{\prime} c^{N}=d^{N}, \\
\mathfrak{Y}=Y \times_{\mathbb{P}^{1}} \mathbb{P}_{\mu}^{1}, & & k b^{N}+k^{\prime} d^{N}=c^{N},
\end{array}
$$

here $[a, c, d],[b, c, d]$ are the homogenous coordinates of $\mathbb{P}^{2}$, which are related with $x, y, x^{\prime}, y^{\prime}, \mu$ by

$$
\left(\frac{a}{d}, \frac{c}{d}\right)=\left(x, \mu^{-1}\right), \quad\left(\frac{b}{c}, \frac{d}{c}\right)=(y, \mu), \quad x^{\prime}=x \mu, \quad y^{\prime}=y \mu^{-1} .
$$

Consider the fiber product of $\mathfrak{X}$ and $\mathfrak{Y}$ over the $\mu$-plane

$$
\mathfrak{W}=\mathfrak{X} \times_{\mathbb{P}_{\mu}^{1}} \mathfrak{Y}\left(=\left(X \times_{\mathbb{P}^{1}} Y\right) \times_{\mathbb{P}^{1}} \mathbb{P}_{\mu}^{1}\right) .
$$

Then $\mathfrak{B}$ is is a curve of genus $N^{3}-2 N^{2}+1$, defined by

$$
\mathfrak{B}:\left\{\begin{array}{l}
k a^{N}+k^{\prime} c^{N}=d^{N} \\
k b^{N}+k^{\prime} d^{N}=c^{N}
\end{array} \quad \text { for }[a, b, c, d] \in \mathbb{P}^{3},\right.
$$

which is also equal to

$$
\left\{\begin{array}{l}
a^{N}+k^{\prime} b^{N}=k d^{N} \\
k^{\prime} a^{N}+b^{N}=k c^{N}
\end{array} \quad \text { for }[a, b, c, d] \in \mathbb{P}^{3} .\right.
$$

Then the curves $W, W_{r}, W_{l}, X, X^{\prime}, Y, Y^{\prime}$ are the quotients of $\mathfrak{B}$ by various order $N^{2}$ subgroups of Aut( $\mathfrak{B})$ which are described as follows:

Define

$$
\begin{gathered}
X=\mathfrak{W} /\left\langle\begin{array}{l}
{[a, b, c, d] \leadsto[a, \omega b, c, d]} \\
{[a, b, c, d] \leadsto[a, b, \omega c, d]}
\end{array}\right\rangle, \\
X^{\prime}=\mathfrak{W} /\left\langle\begin{array}{l}
{[a, b, c, d] \leadsto[a, \omega b, c, d]} \\
{[a, b, c, d] \leadsto[\omega a, b, \omega c, d]}
\end{array}\right\rangle, \\
Y=\mathfrak{W} /\left\langle\begin{array}{l}
{[a, b, c, d] \leadsto[\omega a, b, c, d]} \\
{[a, b, c, d] \leadsto[a, b, c, \omega d]}
\end{array}\right\rangle, \\
Y^{\prime}=\mathfrak{M} /\left\langle\begin{array}{l}
{[a, b, c, d] \leadsto[\omega a, b, c, d]} \\
{[a, b, c, d] \leadsto[a, \omega b, c, \omega d]}
\end{array}\right) .
\end{gathered}
$$

$\mathfrak{J}: \mathfrak{M} \rightarrow \mathfrak{M}, \quad[a, b, c, d] \leadsto[b, a, d, c]$,

$\mathfrak{I}_{\mathbf{r}}: \mathfrak{W} \rightarrow \mathfrak{M}, \quad[a, b, c, d] \leadsto\left[\omega^{\frac{1}{2}} c, d, a, \omega^{\frac{1}{2}} b\right]$,

$\mathfrak{I}_{l}: \mathfrak{M} \rightarrow \mathfrak{M}, \quad[a, b, c, d] \leadsto\left[\omega^{\frac{1}{2}} d, \omega c, b, \omega^{\frac{1}{2}} a\right]$,

$\mathfrak{R}: \mathfrak{W} \rightarrow \mathfrak{W}, \quad[a, b, c, d] \leadsto[\omega a, b, \omega c, d]$,

$\mathfrak{S}: \mathfrak{W} \rightarrow \mathfrak{W}, \quad[a, b, c, d] \leadsto[\omega a, \omega b, c, d]$,

$\mathfrak{I}: \mathfrak{B} \rightarrow \mathfrak{B}, \quad[a, b, c, d] \leadsto[a, \omega b, \omega c, d]$. 
Then it is easy to see

the order of $\mathfrak{R}, \mathfrak{S}, \mathfrak{I}=N$, the order of $\mathfrak{J}, \mathfrak{J}_{l}, \mathfrak{J}_{r}=2$,

and

$$
\begin{array}{ccc}
\mathfrak{R} \mathfrak{I}=\mathfrak{I}^{-1}, & \mathfrak{S} \mathfrak{I}=\mathfrak{I} \mathfrak{S}, & \mathfrak{I} \mathfrak{I}=\mathfrak{I}^{-1}, \\
\mathfrak{R} \mathfrak{I}_{r}=\mathfrak{I}_{r} \mathfrak{R}, & \mathfrak{S}_{r}=\mathfrak{I}_{r} \mathfrak{S}^{-1}, & \mathfrak{I}_{r}=\mathfrak{I}_{r} \mathfrak{I}^{-1}, \\
\mathfrak{R} \mathfrak{I}_{l}=\mathfrak{I}_{l} \mathfrak{R}^{-1}, & \mathfrak{S}_{l}=\mathfrak{I}_{l} \mathfrak{S}^{-1}, & \mathfrak{I} \mathfrak{I}_{l}=\mathfrak{I}_{l} \mathfrak{I}, \\
\mathfrak{R} \mathfrak{I}_{l} \mathfrak{I}=\mathfrak{I}_{l}, & \mathfrak{S}_{\mathfrak{I}_{r}} \mathfrak{I}_{l}=\mathfrak{I}_{l} \mathfrak{I}_{r}, & \mathfrak{I}_{r} \mathfrak{I}=\mathfrak{I} \mathfrak{I}_{r} .
\end{array}
$$

Hence $\left\langle\mathfrak{R}, \mathfrak{S}, \mathfrak{I}, \mathfrak{I}, \mathfrak{I}_{r}, \mathfrak{I}_{l}\right\rangle$ is a group of automorphisms of $\mathfrak{M}$, and is also equal to $\left\langle\mathfrak{I}, \mathfrak{I}_{r}, \mathfrak{I}_{l}\right\rangle$. It can be shown that the quotients of $\mathfrak{W}$ by various normal subgroups of $\left\langle\mathfrak{J}, \mathfrak{I}_{r}, \mathfrak{J}_{l}\right\rangle$ also give the curves $X \times_{\mathbb{P}^{1}} Y, X^{\prime} \times_{\mathbb{P}^{1}} Y^{\prime}, W, W_{r}, W_{l}$ :

$$
\begin{aligned}
X \times_{\mathbb{P}^{1}} Y & =\mathfrak{W} /\langle\mathfrak{T}\rangle, \\
X^{\prime} \times_{\mathbb{P}^{1}} Y^{\prime} & =\mathfrak{W} /\langle\mathfrak{R}\rangle, \\
W & =\mathfrak{M} /\langle\mathfrak{R}, \mathfrak{I}\rangle, \\
W_{r} & =\mathfrak{W} /\langle\mathfrak{S}, \mathfrak{I}\rangle, \\
W_{l} & =\mathfrak{W} /\langle\mathfrak{R}, \mathfrak{S}\rangle .
\end{aligned}
$$

By Hurwitz's Theorem, $\mathfrak{B}$ is the $N^{2}$-fold unramified cover of $W, W_{r}, W_{l}$. This implies that with the morphisms in (16) (or (18)), $X \times_{\mathbb{P}^{1}} Y$ (or $X^{\prime} \times_{\mathbb{P}^{1}} Y^{\prime}$ respectively) is the $N$-fold unramified cover of $W, W_{r}$ (or $W, W_{l}$ respectively). (We have demonstrated here that the curves $W, W_{r}, W_{l}$ can be constructed from the Fermat curves $\mathfrak{X}$ and $\mathfrak{Y}$. In fact, that is how the $C P N$-curves had originally been derived in the physical literature $[1,12]$. The variables, $a, b, c, d$ are the Boltzmann weights of the statistical model.)

The automorphism group $\left\langle\mathfrak{I}, \mathfrak{I}_{r}, \mathfrak{I}_{l}\right\rangle$ of $\mathfrak{W}$ induces the groups of automorphisms of $W, W_{r}, W_{l}$. In fact, these are the group of all automorphisms for $W, W_{r}$, $W_{l}$ by the following result.

Proposition 6. (i) Under the canonical homomorphism $\left\langle\mathfrak{I}, \mathfrak{I}_{\mathbf{r}}, \mathfrak{I}_{l}\right\rangle \rightarrow \operatorname{Aut}(W)$,

$$
\mathfrak{I}_{W}, \quad \mathfrak{I}_{r} \leadsto l_{W}, \quad \mathfrak{I}_{l} \rightsquigarrow \theta_{W} l_{W} \sigma_{W} .
$$

(ii) Under the canonical homomorphism $\left\langle\mathfrak{I}, \mathfrak{I}_{r}, \mathfrak{I}_{l}\right\rangle \rightarrow \operatorname{Aut}\left(W_{r}\right)$,

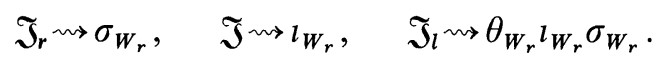

(iii) Under the canonical homomorphism $\left\langle\mathfrak{I}, \mathfrak{I}_{r}, \mathfrak{I}_{l}\right\rangle \rightarrow \operatorname{Aut}\left(W_{l}\right)$,

$$
\mathfrak{I}_{l} \leadsto \sigma_{W_{l}}, \quad \mathfrak{J} \leadsto l_{W_{l}}, \quad \mathfrak{I}_{\mathbf{r}} \leadsto \theta_{W_{l}}^{-1} l_{W_{l}} \sigma_{W_{l}} .
$$

Proof. By (16), (18), (19), (20), (21), $t=\frac{a b}{c d}, \quad t_{r}=\frac{b d}{a c}, \quad t_{l}=\frac{b c}{a d}, \lambda=\left(\frac{d}{c}\right)^{N}$, $\frac{1-k^{\prime} \lambda}{k^{\prime}-\lambda}=-\left(\frac{b}{a}\right)^{N}$. Then the results follows from the the definition of $\mathfrak{I}, \mathfrak{I}_{r}, \mathfrak{I}_{l}$ and (5), (8), (10), (12). q.e.d. 
When $N$ is even, $W_{N, k^{\prime}} /\left\langle\theta^{N / 2}\right\rangle$ is the 1-torus, and it is the double cover of $\mathbb{P}^{1}$ branched at $\left\{0, \infty, k^{\prime}, k^{\prime-1}\right\}$. By Proposition 4 , the 1 -tori determined by $W_{N, k^{\prime}}$, $W_{N, i k^{\prime} / k}, W_{N, k}$ are isomorphic. Hence we have the following diagram:

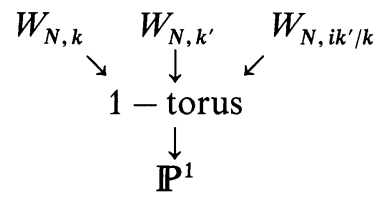

\section{N-Torsion Line Bundles of Chiral Potts $N$-Curves}

As before, $W=W_{N, k^{\prime}}, \mathfrak{W}=$ the fiber product of Fermat curves with the homogeneous coordinate $[a, b, c, d]$ defined in $(21), \mathfrak{R}, \mathfrak{S}, \mathfrak{I}, \mathfrak{I}, \mathfrak{I}_{r}=$ the automorphisms of $\mathfrak{W}$ in (23). Let $\mathbf{u}, \mathbf{u}^{\prime}, \mathbf{v}, \mathbf{v}^{\prime}$ be the elements in $W$ whose $\lambda$-values are $0, \infty, k^{\prime}, k^{\prime-1}$ respectively, and

$$
\mathfrak{p}: \mathfrak{W} \rightarrow W(=\mathfrak{W} /\langle\mathfrak{R}, \mathfrak{T}\rangle)
$$

be the unramified $N^{2}$-fold cover in (25). By (14), (15), (16), (19), (20), the divisors for the sections $a, b, c, d$ in $\mathfrak{M}$ are

$$
\operatorname{div}(a)=\mathfrak{p}^{*}(\mathbf{v}), \quad \operatorname{div}(b)=\mathfrak{p}^{*}\left(\mathbf{v}^{\prime}\right), \quad \operatorname{div}(c)=\mathfrak{p}^{*}\left(\mathbf{u}^{\prime}\right), \quad \operatorname{div}(d)=\mathfrak{p}^{*}(\mathbf{u}) .
$$

For $(m, n) \in(\mathbb{Z} / N \mathbb{Z})^{2}$, let $\kappa_{(m, n)}:\langle\mathfrak{I}, \mathfrak{R}\rangle \rightarrow \mathbb{C}^{\star}$ be the character with $\kappa_{(m, n)}(\mathfrak{I})=\omega^{m}, \kappa_{(m, n)}(\mathfrak{R})=\omega^{n}$. Then

$$
(\mathfrak{B} \times \mathbb{C}) /\left(w \times \zeta \sim w g \times \kappa_{(m, n)}(g) \zeta\right)
$$

is a line bundle over $W$, and its associated $\mathcal{O}_{W}$-sheaf is denoted by $\mathscr{L}_{(m, n)}$.

Proposition 7. (i) $\mathfrak{p}_{*} \mathcal{O}_{\mathfrak{W}}=\bigoplus_{(m, n) \in(\mathbb{Z} / N \mathbb{Z})^{2}} \mathscr{L}_{(m, n)}$.

(ii) $\mathscr{L}_{(1,0)}=\mathcal{O}_{W}\left[\mathbf{v}^{\prime}-\mathbf{u}\right], \mathscr{L}_{(0,1)}=\mathcal{O}_{W}[\mathbf{v}-\mathbf{u}], \mathscr{L}_{(1,1)}=\mathcal{O}_{W}\left[\mathbf{u}^{\prime}-\mathbf{u}\right]$.

Proof. For an open set $U$ of $W$, any function $f$ of $\mathfrak{p}^{-1}(U)$ can be uniquely written as $f=\sum_{(m, n)} f_{(m, n)}$, where $f_{(m, n)}$ is an eigenfunction of $\langle\mathfrak{T}, \mathfrak{R}\rangle$ with eigenvalue $\mathscr{L}_{(m, n)}$. Hence we obtain (i).

With the homogeneous coordinate $[a, b, c, d]$ of $\mathfrak{B}$, the divisors of the rational functions $a / d, b / d, c / d$ of $\mathfrak{M}$ equal to $\mathfrak{p}^{*}(\mathbf{v}-\mathbf{u}), \mathfrak{p}^{*}\left(\mathbf{v}^{\prime}-\mathbf{u}\right), \mathfrak{p}^{*}\left(\mathbf{u}^{\prime}-\mathbf{u}\right)$ respectively. Since the rational function $a / d(b / d, c / d)$ corresponds to a meromorphic section of $\mathscr{L}_{(0,1)}\left(\mathscr{L}_{1,0)}, \mathscr{L}_{(1,1)}\right.$ respectively) over $W$, (ii) follows immediately. q.e.d.

By the same argument, we have the similar conclusions for the curves $W_{r}, W_{l}$.

Proposition 8. Let

and

$$
\mathfrak{p}_{r}: \mathfrak{W} \rightarrow W_{r}(=\mathfrak{W} /\langle\mathfrak{\subseteq}, \mathfrak{x}\rangle)
$$

$$
\mathfrak{p}_{l}: \mathfrak{W} \rightarrow W_{l}\left(=\left.\mathfrak{W}\right|_{\langle\mathfrak{R}, \Im\rangle}\right)
$$


be the unramified $N^{2}$-fold covers in (25). Let $\mathbf{u}_{r}, \mathbf{u}_{r}^{\prime} \mathbf{v}_{r}, \mathbf{v}_{r}^{\prime},\left(\mathbf{u}_{l}, \mathbf{u}_{l}^{\prime}, \mathbf{v}_{l}, \mathbf{v}_{l}^{\prime}\right)$, be the elements of $W_{r},\left(W_{l}\right.$ respectively), such that as the divisors of $\mathfrak{M}$,

$$
\begin{array}{llll}
\operatorname{div}(a)=\mathfrak{p}_{r}^{*}\left(\mathbf{v}_{r}\right), & \operatorname{div}(b)=\mathfrak{p}_{r}^{*}\left(\mathbf{u}_{r}^{\prime}\right), & \operatorname{div}(c)=\mathfrak{p}_{r}^{*}\left(\mathbf{v}_{r}^{\prime}\right), & \operatorname{div}(d)=\mathfrak{p}_{r}^{*}\left(\mathbf{u}_{r}\right), \\
\operatorname{div}(a)=\mathfrak{p}_{l}^{*}\left(\mathbf{u}_{l}^{\prime}\right), & \operatorname{div}(b)=\mathfrak{p}_{l}^{*}\left(\mathbf{v}_{l}\right), & \operatorname{div}(c)=\mathfrak{p}_{l}^{*}\left(\mathbf{v}_{l}^{\prime}\right), & \operatorname{div}(d)=\mathfrak{p}_{l}^{*}\left(\mathbf{u}_{l}\right) .
\end{array}
$$

\section{Denote}

$$
\begin{gathered}
\mathscr{L}_{r(m, n)}=\text { the } \mathcal{O}_{W_{r}} \text {-sheaf associated the character }\langle\mathfrak{S}, \mathfrak{I}\rangle \rightarrow \mathbb{C}^{\star}, \\
\qquad \mathfrak{I} \rightsquigarrow \omega^{m,} \quad \mathfrak{S} \rightsquigarrow \omega^{n} . \\
\mathscr{L}_{l(m, n)}=\text { the } \mathcal{O}_{W_{l}} \text {-sheaf associated to the character }\langle\mathfrak{R}, \mathfrak{S}\rangle \rightarrow \mathbb{C}^{\star}, \\
\mathfrak{R} \rightsquigarrow \omega^{m,} \quad \mathbb{S} \leadsto \omega^{n} .
\end{gathered}
$$

Then

(i) $\mathfrak{p}_{r *} \mathcal{O}_{\mathfrak{B}}=\bigoplus_{(m, n) \in(\mathbb{Z} / N \mathbb{Z})^{2}} \mathscr{L}_{r(m, n)}, \quad \mathfrak{p}_{l *} \mathcal{O}_{\mathfrak{B}}=\bigoplus_{(m, n) \in(\mathbb{Z} / N \mathbb{Z})^{2}} \mathscr{L}_{l(m, n)}$.

(ii) Over the curves $W_{r}, W_{l}$, we have

$$
\begin{array}{lll}
\mathscr{L}_{r(1,0)}=\mathcal{O}_{W_{r}}\left[\mathbf{v}_{r}^{\prime}-\mathbf{u}_{r}\right], & \mathscr{L}_{r(0,1)}=\mathcal{O}_{W_{r}}\left[\mathbf{v}_{r}-\mathbf{u}_{r}\right], & \mathscr{L}_{r(1,1)}=\mathcal{O}_{W_{r}}\left[\mathbf{u}_{r}^{\prime}-\mathbf{u}_{r}\right], \\
\mathscr{L}_{l(1,0)}=\mathcal{O}_{W_{l}}\left[\mathbf{v}_{l}^{\prime}-\mathbf{u}_{l}\right], & \mathscr{L}_{l(0,1)}=\mathcal{O}_{W_{l}}\left[\mathbf{v}_{l}-\mathbf{u}_{l}\right], & \mathscr{L}_{l(1,1)}=\mathcal{O}_{W_{l}}\left[\mathbf{u}_{l}^{\prime}-\mathbf{u}_{l}\right] .
\end{array}
$$

\section{Jacobian Variety of a Chiral Potts $N$-Curve}

Let $r$ be an element in $\mathbb{C}-\{0, \pm 1\}, \omega=e^{2 \pi i / N}, g=N-1$. Assume that $\left|\frac{1-r}{1+r}\right| \leqq 1$, and denote $\left(\frac{1-r}{1+r}\right)^{1 / N}=\left|\frac{1-r}{1+r}\right|^{1 / N} e^{i \vartheta / N}$; here $0 \leqq \vartheta=\arg \left(\frac{1-r}{1+r}\right)<2 \pi$. In this section, we shall denote

$$
W=W_{N, r},
$$

$\Psi, \psi, \Pi, \pi=$ the morphisms of (3) for $W$,

$$
\{\theta, \sigma, l\}=\text { the generators of } \operatorname{Aut}(W) \text { in Proposition } 5 \text {. }
$$

$W$ is a genus $g \mathrm{CP} N$-curve defined by the coordinate $(t, \lambda)$ of $(4)$, or $(t, w)$ of $(7)$. Denote

$$
\operatorname{Jac}(W)=\text { the Jacobian variety of } W \text {. }
$$

The critical points of $\Pi$ are the elements $\mathbf{b}_{j}, \mathbf{b}_{j}^{\prime}, 1 \leqq j \leqq N$, of $W$ with $\Pi\left(\mathbf{b}_{j}\right)=\omega^{-j}\left(\frac{1+r}{1-r}\right)^{1 / N}$ and $\Pi\left(\mathbf{b}_{j}^{\prime}\right)=\omega^{-j}\left(\frac{1-r}{1+r}\right)^{1 / N}$. We also denote $\mathbf{o}=\mathbf{b}_{N}$, $\mathbf{o}^{\prime}=\mathbf{b}_{N}^{\prime}$. The critical points of $\Psi$ are the elements $\mathbf{p}, \mathbf{p}^{\prime}, \mathbf{q}, \mathbf{q}^{\prime}$ of $W$ with the $\lambda$-values $0, \infty, r, r^{-1}$ respectively. Then $\mathbf{p}$ and $\mathbf{q}$ are on the "same sheet" of the covering of $\Pi$. (Here we consider $W$ as the double cover over the $t$-plane with cuts on the segments from $\omega^{j}\left(\frac{1-r}{1+r}\right)^{1 / N}$ to $\omega^{j}\left(\frac{1+r}{1-r}\right)^{1 / N}, 1 \leqq j \leqq N$. The value of the coordinate $w$ determine the "sheet" of $W$.) We are going to describe Jac $(W)$ using the data of $\left\{\mathbf{b}_{j}, \mathbf{b}_{j}^{\prime} \mid 1 \leqq j \leqq N\right\}$, and then $\left\{\mathbf{p}, \mathbf{p}^{\prime}, \mathbf{q}, \mathbf{q}^{\prime}\right\}$.

It is well-known that the abelian differentials of first kind for $W$ has the following expression: 
Lemma 5. $\Gamma\left(W, \Omega^{1}\right)=$ the $\mathbb{C}$-space consisting of

$$
p(t) \frac{d t}{w}, \quad p(t)=\text { a polynomial of degree } \leqq(N-2) .
$$

Denote $\varphi_{\alpha}=t^{\alpha-1} \frac{d t}{w}$ for $1 \leqq \alpha \leqq g$. Then $\left\{\varphi_{\alpha}, 1 \leqq \alpha \leqq g\right\}$ forms a base of $I\left(W, \Omega^{1}\right)$ with

$$
\theta^{*}\left(\varphi_{\alpha}\right)=\omega^{\alpha} \varphi_{\alpha}, \quad \sigma^{*}\left(\varphi_{\alpha}\right)=-\varphi_{\alpha}, \quad l^{*}\left(\varphi_{\alpha}\right)=-\varphi_{N-\alpha} .
$$

By a path in $W$ we shall always mean an oriented one. It is easy to see the following lemma holds.

Lemma 6. For a path $\gamma$ in $W, \underset{\theta^{m}(\gamma)}{ } \varphi_{\alpha}=\omega^{\alpha m} \int_{\gamma} \varphi_{\alpha}$.

We shall denote

$$
\int_{\gamma} \varphi=\left(\begin{array}{c}
\int_{\gamma} \varphi_{1} \\
\vdots \\
\int_{\gamma} \varphi_{g}
\end{array}\right) .
$$

Let $A$ and $B$ be the $\sigma$-invariant 1 -cycles on $W$ such that by the morphism $\Pi, A$ lies over the segment from $\left(\frac{1-r}{1+r}\right)^{1 / N}$ to $\left(\frac{1+r}{1-r}\right)^{1 / N}$, and $B$ lies over a path from $\omega\left(\frac{1-r}{1+r}\right)^{1 / N}$ to $\left(\frac{1+r}{1-r}\right)^{1 / N}$ with $A$ intersecting $B$ only at $\mathrm{o}$ having the intersection number $I(A, B)=1$. Define

$$
\begin{gathered}
A_{j}=\theta^{-j}(A), \quad B_{j}=\theta^{-j}(B) \text { for integer } j . \\
C_{\alpha}=\sum_{1 \leqq j \leqq \alpha} B_{j} \text { for } \quad 1 \leqq \alpha \leqq g .
\end{gathered}
$$

Then $A_{j+N}=A_{j}, B_{j+N}=B_{j}$, and $\sum_{1 \leqq j \leqq N} A_{j}, \sum_{1 \leqq j \leqq N} B_{j}$ are homologous to zero.

(The cycles $A_{\alpha}, B_{\alpha}, C_{\alpha}$ are shown in Fig. 1.)

The intersection numbers of the cycles $A_{\alpha}, B_{\alpha}, C_{\alpha}, 1 \leqq \alpha \leqq g$, are given by

$$
\begin{gathered}
I\left(A_{\alpha}, A_{\beta}\right)=I\left(B_{\alpha}, B_{\beta}\right)=0, \\
I\left(A_{\alpha}, B_{\beta}\right)=0, \quad \text { except } I\left(A_{\alpha}, B_{\alpha}\right)=1, \quad I\left(A_{\alpha}, B_{\alpha+1}\right)=-1 .
\end{gathered}
$$

Hence

$$
I\left(A_{\alpha}, A_{\beta}\right)=I\left(C_{\alpha}, C_{\beta}\right)=0, \quad I\left(A_{\alpha}, C_{\beta}\right)=\delta_{\alpha \beta} .
$$

Let

$$
l=\int_{A} \varphi=\left(\begin{array}{c}
l_{1} \\
\vdots \\
l_{g}
\end{array}\right), \quad l^{\prime}=\int_{B} \varphi=\left(\begin{array}{c}
l_{1}^{\prime} \\
\vdots \\
l_{g}^{\prime}
\end{array}\right) .
$$




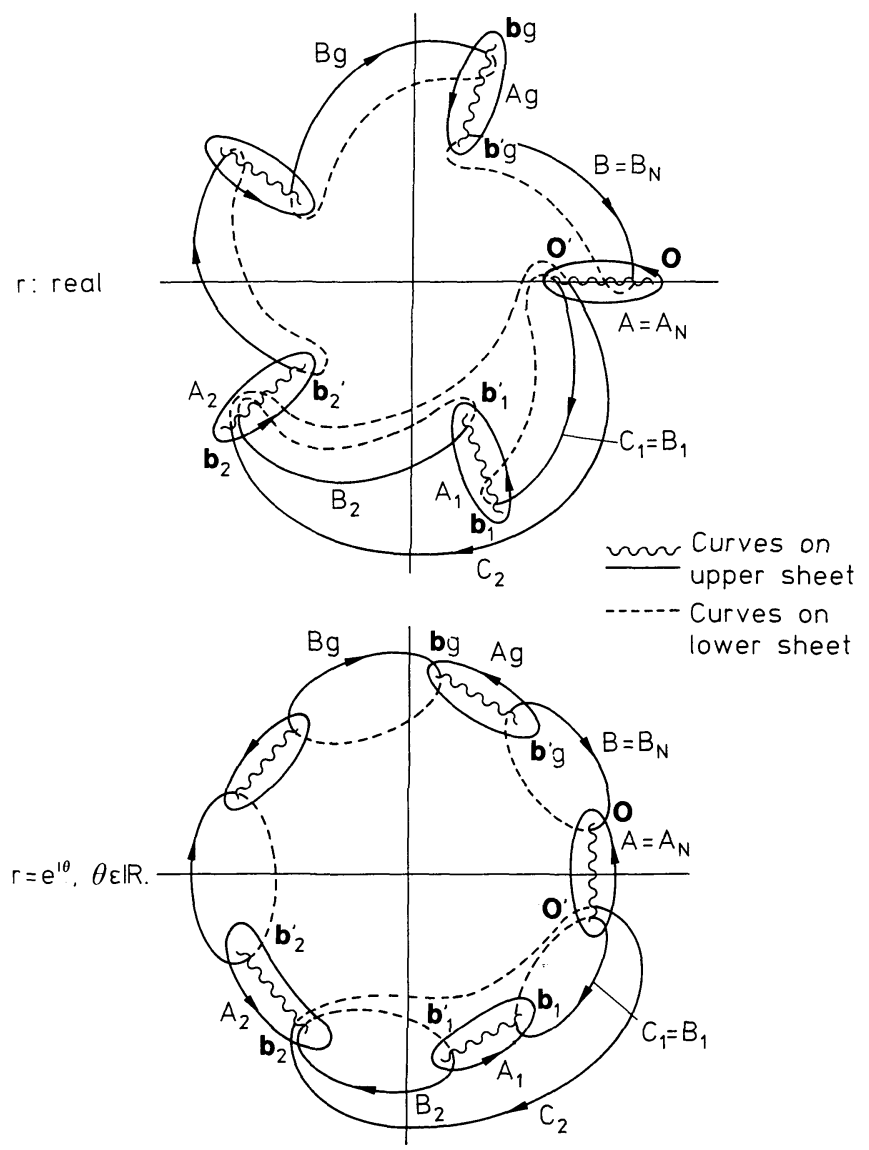

Fig. 1. The curves homologous to $A_{j}, B_{j}, C_{j}$ for $N=5$

By Lemma 6,

$$
\begin{aligned}
& \left(\int_{A_{1}} \varphi_{r} \ldots, \int_{A_{g}} \varphi\right)=\left(\delta_{\alpha \beta} l_{\alpha}\right)\left(\omega^{-\alpha \beta}\right)_{1 \leqq \alpha, \beta \leqq g} . \\
& \left(\int_{B_{1}} \varphi_{r} \ldots, \int_{B_{g}} \varphi\right)=\left(\delta_{\alpha \beta} l_{\alpha}^{\prime}\right)\left(\omega^{-\alpha \beta}\right)_{1 \leqq \alpha, \beta \leqq g} .
\end{aligned}
$$

Hence

$$
\left(\int_{C_{1}} \varphi, \ldots, \int_{C_{g}} \varphi\right)=\left(\delta_{\alpha \beta} l_{\alpha}^{\prime}\right)\left(k_{\alpha \beta}^{\prime}\right)_{1 \leqq \alpha, \beta \leqq g} .
$$

here $k_{\alpha \beta}^{\prime}=\sum_{1 \leqq m \leqq \beta} \omega^{-m \alpha}=i e^{\pi i / N}\left(\omega^{-\alpha \beta}-1\right) / 2 \sin \frac{\pi \alpha}{N}$.

Let $\phi_{1}, \ldots, \phi_{g}$ the base of $\Gamma\left(W, \Omega^{1}\right)$ defined by

$$
\phi=\left(\begin{array}{c}
\phi_{1} \\
\vdots \\
\phi_{g}
\end{array}\right)=\left(\omega^{-\alpha \beta}\right)^{-1}\left(\delta_{\alpha \beta} l_{\alpha}\right)^{-1}\left(\begin{array}{c}
\varphi_{1} \\
\vdots \\
\varphi_{g}
\end{array}\right),
$$


then

$$
\left(\int_{A_{\alpha}} \phi, \int_{B_{\alpha}} \phi\right)=\left(I_{g},\left(\tau_{\alpha \beta}\right)\right)
$$

Here

$$
\begin{gathered}
\tau_{\alpha \beta}=\frac{i}{2 N} \sum_{\gamma} \frac{\left(\omega^{\alpha \gamma}-1\right)\left(\omega^{-\gamma \beta}-1\right) \omega^{-\gamma / 2}}{\sin \frac{\pi \gamma}{N}} \chi_{\gamma} \\
=\frac{2 i}{N} \sum_{\gamma} \frac{\cos \frac{\pi \gamma(\alpha-\beta)}{N} \sin \frac{\pi \alpha \gamma}{N} \sin \frac{\pi \beta \gamma}{N} \omega^{-\gamma / 2}}{\sin \frac{\pi \gamma}{N}} \chi_{\gamma} \\
\text { with } \chi_{\gamma}=\frac{l_{\gamma}^{\prime}}{l_{\gamma}}
\end{gathered}
$$

(The above $\omega^{-\gamma / 2} \chi_{\gamma}$ for $W=W_{N, i k^{\prime} / k}$ is equal to the $\frac{L_{\gamma}^{\prime}}{L_{\gamma}}$ in [6].)

Hence the period of the Jacobian of $W$ is equal to $\tau={ }^{t} \tau=\left(\tau_{\alpha \beta}\right)_{1 \leqq \alpha, \beta \leqq g}$ and

$$
\operatorname{Jac}(W)=\text { the torus } \mathbb{C}^{g} /\left(\mathbb{Z}^{g}+\tau \mathbb{Z}^{g}\right) .
$$

For a fixed base point $\star$ of $W$, we have the canonical embedding of $W$ into $\operatorname{Jac}(W)$ :

$$
\begin{aligned}
& W \rightarrow \operatorname{Jac}(W), \\
& w \leadsto\left[\int_{\star}^{w} \phi\right] .
\end{aligned}
$$

The period of Jac $(W)$ is related to the elements $\mathbf{b}_{j}, \mathbf{b}_{j}^{\prime}$ by the following proposition.

Proposition 8. For $1 \leqq \beta \leqq g$, let

$$
\begin{aligned}
\tau_{\beta} & =\text { the } \beta^{\text {th }} \text { column of }\left(\tau_{\alpha \beta}\right), \\
e_{\beta} & =\text { the } \beta^{\text {th }} \text { element of the standard base of } \mathbb{Z}^{g} .
\end{aligned}
$$

Denote $\frac{1}{2}=\frac{1}{2} \sum_{1 \leqq \beta \leqq g} e_{\beta}$.

(i) For an element $\mathbf{w}$ of $W$, let $[s],[z]$ be the images of $\mathbf{w}$ under the maps (29) for $\star=\mathbf{0}, \mathbf{o}^{\prime}$ respectively. Then $\left[s-\frac{1}{2}\right]=[z]$.

(ii) Under the map (29) for $\star=\mathbf{o}^{\prime}$,

$$
\begin{aligned}
& \mathbf{o} \leadsto\left[\frac{1}{2}\right], \\
& \mathbf{b}_{\beta} \leadsto\left[\frac{1}{2} \tau_{\beta}+\frac{1}{2} \sum_{\alpha \leqq(\beta-1)} e_{\alpha}\right], \\
& \mathbf{b}_{\beta}^{\prime} \leadsto\left[\frac{1}{2} \tau_{\beta}+\frac{1}{2} \sum_{\alpha \leqq \beta} e_{\alpha}\right], \quad \text { for } 1 \leqq \beta \leqq g .
\end{aligned}
$$


Proof. Let $\overline{\mathbf{o o}^{\prime}}$ be the path from $\mathbf{o}$ to $\mathbf{o}^{\prime}$ along the curve $A$. Then

$$
\begin{aligned}
& \underset{\underline{\mathbf{o o}^{\prime}}}{\int} \phi=\frac{1}{2}\left(\int_{\underline{\mathbf{o o}^{\prime}}} \phi+\int_{-\sigma\left(\overline{\mathbf{o o}^{\prime}}\right)} \phi\right) \\
& =\frac{1}{2} \int_{A} \phi=\frac{1}{2} \int_{\substack{\Sigma \\
1 \leqq \alpha \leqq g}} \phi=-\frac{1}{2} \text {. }
\end{aligned}
$$

Hence

$$
[z]=\left[\int_{\mathbf{o}^{\prime}}^{w} \phi\right]=\left[\int_{\mathbf{o}}^{w} \phi\right]-\left[\int_{\frac{\mathbf{o o}^{\prime}}{}} \phi\right]=\left[s-\frac{1}{2}\right] .
$$

So we obtain (i), hence the first correspondence of (ii). Let $\overline{\mathbf{b}_{j-1}^{\prime} \mathbf{b}_{j}},\left(\overline{\mathbf{b}_{j} \mathbf{b}_{j}^{\prime}}\right)$, $1 \leqq j \leqq N$, be the path from $\mathbf{b}_{j-1}^{\prime}$ to $\mathbf{b}_{j}\left(\mathbf{b}_{j}\right.$ to $\mathbf{b}_{j}^{\prime}$ respectively) along $B_{j}\left(A_{j}\right.$ respectively). Let $\overline{\mathbf{o}^{\prime} \mathbf{b}_{\beta}}, \overline{\mathbf{o}^{\prime} \mathbf{b}_{\beta}^{\prime}}, 1 \leqq \beta \leqq g$, be the curves from $\mathbf{o}^{\prime}$ to $\mathbf{b}_{\beta}, \mathbf{b}_{\beta}^{\prime}$ defined by

$$
\begin{aligned}
& \overline{\mathbf{o}^{\prime} \mathbf{b}_{\beta}}=\sum_{1 \leqq j \leqq \beta} \overline{\mathbf{b}_{j-1}^{\prime} \mathbf{b}_{j}}+\sum_{1 \leqq j \leqq(\beta-1)} \overline{\mathbf{b}_{j} \mathbf{b}_{j}^{\prime}}, \\
& \overline{\mathbf{0}^{\prime} \mathbf{b}_{\beta}^{\prime}}=\overline{\mathbf{o}^{\prime} \mathbf{b}_{\beta}}+\overline{\mathbf{b}_{\beta} \mathbf{b}_{\beta}^{\prime}} .
\end{aligned}
$$

Then

$$
\begin{aligned}
& \underset{\frac{\mathbf{b}_{J} \mathbf{b}_{j}^{\prime}}{2}}{\int} \int_{A_{J}} \phi=\frac{-1}{2} e_{j}, \\
& \int_{\substack{\Sigma \leqq j \leqq \beta \\
\overline{\mathbf{b}_{j}^{\prime}-1} \mathbf{b}_{j}}} \phi=\frac{1}{2} \int_{\substack{\Sigma \\
1 \leqq j \leqq \beta}} \phi=\frac{1}{2} \int_{C_{\beta}} \phi=\frac{1}{2} \tau_{\beta}, \\
& \underset{\mathbf{o}^{\prime} \mathbf{b}_{\beta}}{\int} \phi=\frac{1}{2} \tau_{\beta}-\frac{1}{2} \sum_{j \leqq(\beta-1)} e_{j}, \\
& \underset{\mathbf{o}^{\prime} \mathbf{b}_{\beta}^{\prime}}{\int_{1}} \phi=\frac{1}{2} \tau_{\beta}-\frac{1}{2} \sum_{j \leqq \beta} e_{j} .
\end{aligned}
$$

Hence we obtain (ii). q.e.d.

We are going to express the period of $\operatorname{Jac}(W)$ in terms of the data $\left\{\mathbf{p}, \mathbf{p}^{\prime}, \mathbf{q}, \mathbf{q}^{\prime}\right\}$. Define

$$
\begin{gathered}
\varepsilon=\left[\begin{array}{c}
\varepsilon_{1} \\
\varepsilon_{2} \\
\vdots \\
\varepsilon_{g}
\end{array}\right]=\frac{1}{N}\left[\begin{array}{c}
1 \\
2 \\
\vdots \\
g
\end{array}\right], \\
\varrho=\left(\begin{array}{c}
\varrho_{1} \\
\vdots \\
\varrho_{g}
\end{array}\right)=\frac{1}{N}\left(\begin{array}{c}
\sum_{1 \leqq \beta \leqq g} \tau_{1 \beta} \\
\vdots \\
\sum_{1 \leqq \beta \leqq g} \tau_{g \beta}
\end{array}\right) .
\end{gathered}
$$


Consider the following paths in $W$ :

$\overline{\mathbf{o \mathbf { q } ^ { \prime }}}\left(\overline{\mathbf{o}^{\prime} \mathbf{q}^{\prime}}\right)=$ the path from $\mathbf{o}\left(\mathbf{o}^{\prime}\right.$ respectively) to $\mathbf{q}^{\prime}$ with $t$-values in the segment from $\left(\frac{1+r}{1-r}\right)^{1 / N}\left(\left(\frac{1-r}{1+r}\right)^{1 / N}\right.$ respectively $)$ to 0 ,

$$
\begin{gathered}
\overline{\mathbf{p o}}=\imath\left(-\overline{\mathbf{o}^{\prime} \mathbf{q}^{\prime}}\right), \\
\overline{\mathbf{p \mathbf { p } ^ { \prime }}}=\overline{\mathbf{p o}}-\sigma(\overline{\mathbf{p o}}), \quad \overline{\mathbf{p q ^ { \prime }}}=\overline{\mathbf{p o}}+\overline{\mathbf{o q ^ { \prime }}} . \\
\overline{\mathbf{p q}}=\overline{\mathbf{p o}}+\sigma(\overline{\mathbf{o q}}), \quad \overline{\mathbf{p q}^{\prime} \mathbf{q}^{\prime}}=\sigma(\overline{\mathbf{p q}}) .
\end{gathered}
$$

(Then above paths are shown in Fig. 2.) Then

$$
\begin{gathered}
\theta^{j-1}\left(\overline{\mathbf{p}^{\prime} \mathbf{q}^{\prime}}\right)-\theta^{j}\left(\overline{\mathbf{p}^{\prime} \mathbf{q}^{\prime}}\right) \text { is homologous to }-A_{N-j}, \\
\theta^{-j+1}\left(\overline{\mathbf{p p}^{\prime}}\right)-\theta^{-j}\left(\overline{\mathbf{p p}^{\prime}}\right)=-A_{j-1}+B_{j} .
\end{gathered}
$$

Lemma 7. $\underset{\mathbf{p}^{\prime} \mathbf{q}^{\prime}}{ } \phi=-\varepsilon, \quad \frac{\int}{\mathbf{p \mathbf { p } ^ { \prime }}} \phi=\varrho+\varepsilon, \underset{\frac{\mathbf{p q}^{\prime}}{}}{ } \phi=\varrho, \underset{\frac{\mathbf{p q}}{\mathbf{p}}}{ } \phi=\varepsilon$.

Proof. For $0 \leqq j \leqq N-1$, we have

$$
\begin{gathered}
\left(\omega^{-\alpha \beta}\right)^{-1}\left(\delta_{\alpha \beta} l_{\alpha}\right)^{-1} \int_{\theta^{j}\left(\overline{\left.\mathbf{p}^{\prime} \mathbf{q}^{\prime}\right)}\right.} \varphi=\int_{\theta^{j}\left(\overline{\mathbf{p}^{\prime} \mathbf{q}^{\prime}}\right)} \phi=\int_{\overline{\mathbf{p}^{\prime} \mathbf{q}^{\prime}}} \phi+\int_{(N-1) \geqq m \geqq(N-j)} \int_{A_{m}} \phi, \\
\left(\omega^{-\alpha \beta}\right)^{-1}\left(\delta_{\alpha \beta} l_{\alpha}\right)^{-1} \int_{\theta^{-j}\left(\overline{\mathbf{p}^{\prime}}\right)} \varphi=\int_{\theta^{-i}\left(\overline{\mathbf{p p}^{\prime}}\right)} \phi=\int_{\overline{\mathbf{p}^{\prime}}} \phi-\int_{C_{j}} \phi+\int_{(j-1) \geqq m \geqq 0} \int_{A_{m}} \phi,
\end{gathered}
$$

(here $C_{0}=\sum_{N \geqq m \geqq 1} B_{m}$ which is homologous to 0 ).

By Lemma 6 ,

$$
\begin{aligned}
0 & =\sum_{(N-1) \geqq j \geqq 0}\left[\left(\omega^{-\alpha \beta}\right)^{-1}\left(\delta_{\alpha \beta} l_{\alpha}\right)^{-1} \int_{\theta^{J}\left(\overline{\mathbf{p}^{\prime} \mathbf{q}^{\prime}}\right)} \varphi\right]=N \int_{\overline{\mathbf{p}^{\prime} \mathbf{q}^{\prime}}} \phi+\int_{(N-1) \geqq m \geqq 1}^{\int_{m A_{m}} \phi,} \\
0 & =\sum_{(N-1) \geqq j \geqq 0}\left[\left(\omega^{-\alpha \beta}\right)^{-1}\left(\delta_{\alpha \beta} l_{\alpha}\right)^{-1} \int_{\theta^{-j}\left(\overline{\mathbf{p} \bar{p}^{\prime}}\right)} \varphi\right] \\
& =N \int_{\mathbf{p p}^{\prime}} \phi-\int_{(N-1) \geqq j \geqq 1} \int_{C_{j}} \phi-\int_{(N-1) \geqq m \geqq 1}^{\Sigma} \int_{m A_{m}} \phi,
\end{aligned}
$$

which imply the first two equalities, then the rest follows immediately. q.e.d.

Proposition 9. Under the map (29) for $\star=\mathbf{0}$,

$$
\begin{array}{ll}
\mathbf{p} \rightsquigarrow\left[\frac{-1}{2} \varrho-\frac{1}{2} \varepsilon\right], & \mathbf{p}^{\prime} \rightsquigarrow\left[\frac{1}{2} \varrho+\frac{1}{2} \varepsilon\right], \\
\mathbf{q} \rightsquigarrow\left[\frac{-1}{2} \varrho+\frac{1}{2} \varepsilon\right], & \mathbf{q}^{\prime} \rightsquigarrow\left[\frac{1}{2} \varrho-\frac{1}{2} \varepsilon\right] .
\end{array}
$$



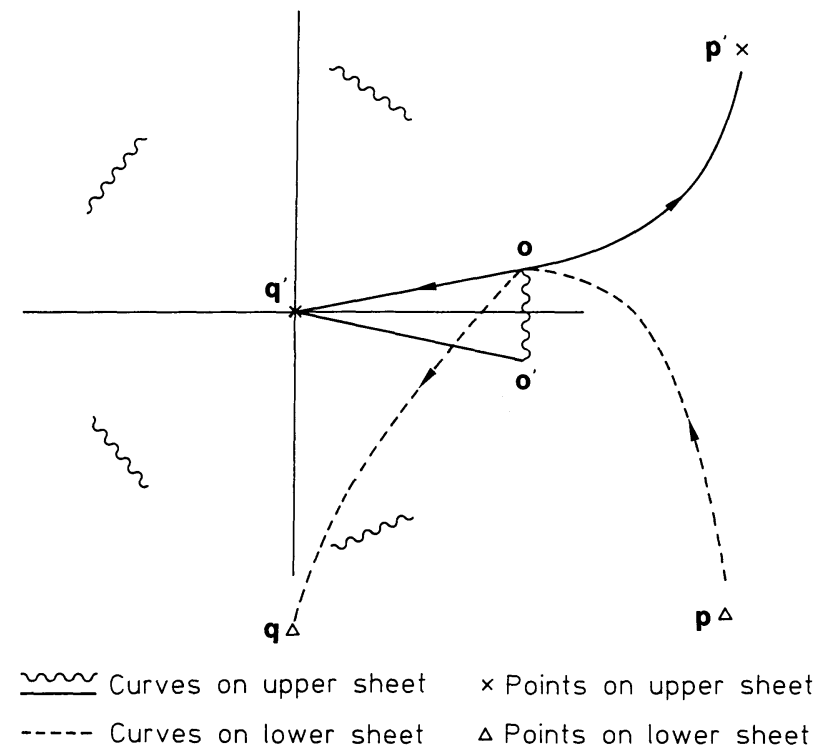

Fig. 2. The paths pq, $\mathbf{p q}{ }^{\prime}, \mathbf{p p} \mathbf{p}^{\prime}$ for $N=5, r=e^{i \theta}(\theta \in \mathbb{R})$

Proof. We have

$$
\begin{aligned}
& \int_{\mathbf{0}}^{\mathbf{p}} \phi=\frac{-1}{2}\left(\int_{\overline{\mathbf{p o}}} \phi+\int_{\sigma(\overline{\mathbf{p o}})} \sigma^{*} \phi\right) \\
& =\frac{-1}{2} \underset{\overline{\mathbf{p o}}-\sigma(\overline{\mathbf{p o}})}{ } \phi=\frac{-1}{2} \int_{\frac{\mathbf{p p}^{\prime}}{} \phi} \phi \\
& =\frac{-1}{2} \varrho-\frac{1}{2} \varepsilon, \quad(\text { by Lemma 7) }
\end{aligned}
$$

Hence we obtain the value of $\mathbf{p}$ of the map (29) for $\star=\mathbf{o}$. The other values of this map follow from Lemma 7 and the following identities:

$$
\begin{aligned}
\int_{\mathbf{o}}^{\mathbf{p}^{\prime}} \phi & =\int_{\mathbf{o}}^{\mathbf{p}} \phi+\int_{\frac{\mathbf{p} \mathbf{p}^{\prime}}{}} \phi^{\prime} \\
\int_{\mathbf{o}}^{\mathbf{q}} \phi & =\int_{\mathbf{0}}^{\mathbf{p}} \phi+\int_{\frac{\mathbf{p q}}{} \phi,} \phi \\
\int_{\mathbf{0}}^{\mathbf{q}^{\prime}} \phi & =\int_{\mathbf{0}}^{\mathbf{p}} \phi+\int_{\frac{\int_{\mathbf{p q}}^{\prime}}{} \phi .}
\end{aligned}
$$

q.e.d.

Lemma 8. For a path $\gamma$ in $W$,

$$
\int_{\theta^{-1}(\gamma)} \phi=\left(r_{\alpha \beta}\right) \int_{\gamma} \phi,
$$


here

$$
r_{\alpha \beta}=0 \text { except } r_{(\beta+1) \beta}=1(1 \leqq \beta \leqq g), r_{\alpha g}=-1(1 \leqq \alpha \leqq g)
$$

Proof. By Lemma 6, we have

$$
\begin{aligned}
\int_{\theta^{-1}(\gamma)} \phi & =\left(\omega^{-\alpha \beta}\right)^{-1}\left(\delta_{\alpha \beta} l_{\alpha}\right)^{-1} \int_{\theta^{-1}(\gamma)} \varphi \\
& =\left(\omega^{-\alpha \beta}\right)^{-1}\left(\delta_{\alpha \beta} l_{\alpha}\right)^{-1}\left(\delta_{\alpha \beta} \omega^{-\alpha}\right) \int_{\gamma} \varphi \\
& =\left(\omega^{-\alpha \beta}\right)^{-1}\left(\delta_{\alpha \beta} l_{\alpha}\right)^{-1}\left(\delta_{\alpha \beta} \omega^{-\alpha}\right)\left(\delta_{\alpha \beta} l_{\alpha}\right)\left(\omega^{-\alpha \beta}\right) \int_{\gamma} \phi \\
& =\left(\omega^{-\alpha \beta}\right)^{-1}\left(\delta_{\alpha \beta} \omega^{-\alpha}\right)\left(\omega^{-\alpha \beta}\right) \int_{\gamma} \phi,
\end{aligned}
$$

which implies the result. q.e.d.

The period of $\operatorname{Jac}(W)$ is expressed by the vector $\varrho$ via the following proposition.

Proposition 10. Define $\varrho_{0}=0$. Then for $\alpha, \beta=1, \ldots g$,

$$
\begin{aligned}
\varrho_{\alpha} & =\varrho_{N-\alpha}, \\
\tau_{\alpha \beta} & =\varrho_{\alpha}+\varrho_{\beta}-\varrho_{|\alpha-\beta|} .
\end{aligned}
$$

Proof. It is easy to see that $-l(A)$ is homologous to $A$. Hence the $l_{\alpha}$ 's in (27) have the relation:

$$
l_{\alpha}=l_{N-\alpha} \text { for } 1 \leqq \alpha \leqq g
$$

By the definition of the paths,

$$
-l\left(\overline{\mathbf{p q}^{\prime}}\right) \text { is homologous to } \overline{\mathbf{p q}} \text {. }
$$

Hence

$$
\begin{aligned}
& \varrho=\int_{\overline{\mathbf{p q}}} \phi=-\int_{\imath\left(\mathbf{p q}^{\prime}\right)} \phi=-\int_{\frac{\mathbf{p q ^ { \prime }}}{}} \imath^{*}(\phi) \\
& =-\left(\omega^{-\alpha \beta}\right)^{-1}\left(\delta_{\alpha \beta} l_{\alpha}\right)^{-1} \frac{\int_{\mathbf{p q}^{\prime}}}{*}(\varphi) \\
& =\left(\omega^{-\alpha \beta}\right)^{-1}\left(\delta_{\alpha \beta} l_{\alpha}\right)^{-1}\left(\delta_{\alpha(N-\alpha)}\right) \int_{\frac{\mathbf{p q}}{\prime}} \varphi \\
& =\left(\omega^{-\alpha \beta}\right)^{-1}\left(\delta_{\alpha \beta} l_{\alpha}\right)^{-1}\left(\delta_{\alpha(N-\alpha)}\right)\left(\delta_{\alpha \beta} l_{\alpha}\right)\left(\omega^{-\alpha \beta}\right) \underset{\frac{\mathbf{p} \mathbf{q}^{\prime}}{} \phi}{ } \phi \\
& =\left(\omega^{-\alpha \beta}\right)^{-1}\left(\delta_{\alpha \beta} l_{\alpha}\right)^{-1}\left(\delta_{\alpha \beta} l_{\alpha}\right)\left(\delta_{\alpha(N-\alpha)}\right)\left(\omega^{-\alpha \beta}\right) \varrho \\
& =\left(\omega^{-\alpha \beta}\right)^{-1}\left(\delta_{\alpha(N-\alpha)}\right)\left(\omega^{-\alpha \beta}\right)\left(\delta_{\alpha(N-\alpha)}\right)^{2} \varrho=\left(\delta_{\alpha(N-\alpha)}\right) \varrho \text {, }
\end{aligned}
$$

which implies $\varrho_{\alpha}=\varrho_{N-\alpha}$.

We have

$$
\begin{gathered}
\theta^{-\beta+1}\left(\overline{\mathbf{p q}^{\prime}}\right)-\theta^{-\beta}\left(\overline{\mathbf{p q}^{\prime}}\right)=B_{\beta}, \\
\overline{\mathbf{p q}^{\prime}}-\theta^{-\beta}\left(\overline{\mathbf{p q}^{\prime}}\right)=C_{\beta}, \quad \text { for } 1 \leqq \beta \leqq g .
\end{gathered}
$$


Hence

$$
\begin{aligned}
\int_{C_{\beta}} \phi & =\int_{\overline{\mathbf{p q}}} \phi-\int_{\theta^{-\beta}\left(\overline{\mathbf{p q ^ { \prime }}}\right)} \phi \\
& =\varrho-\left(r_{\alpha \gamma}\right)^{\beta} \varrho, \quad(\text { by Lemma 7), }
\end{aligned}
$$

which implies

$$
\tau_{\alpha \beta}=\varrho_{\alpha}+\varrho_{N-\beta}-\varrho_{|\alpha-\beta|}=\varrho_{\alpha}+\varrho_{\beta}-\varrho_{|\alpha-\beta|} \text {. q.e.d. }
$$

Remark. In [6], the $\varrho_{\alpha}$ 's are solved from the expression of $\tau_{\alpha \beta}$, and described by

$$
\varrho_{\alpha}=\frac{i}{N} \sum_{\gamma} \frac{\sin ^{2}\left(\frac{\pi \alpha \gamma}{N}\right)}{\sin \frac{\pi \gamma}{N}}\left(\omega^{-\gamma / 2} \frac{l_{\gamma}^{\prime}}{l_{\gamma}}\right) .
$$

Then the identities in the above proposition can be verified through the explicit expressions of $\varrho_{\alpha}$ and $\tau_{\alpha \beta}$. The proof we give here indicates these identities follow from the symmetries of the CP $N$-curve $W$.

By the uniformizing coordinate of $\operatorname{Jac}(W)$,

$$
s=\left(\begin{array}{c}
s_{1} \\
\vdots \\
s_{g}
\end{array}\right) \in \mathbb{C}^{g},
$$

the hyperelliptic theta function of $\operatorname{Jac}(W)$ is

$$
\Theta(s)(=\vartheta(s, \tau))=\sum_{m \in \mathbb{Z}^{g}} e^{2 \pi i^{t} m s+\pi i^{t} m r m},
$$

and by Proposition 10 it can also be written as

$$
\Theta(s)=\sum_{m \in \mathbb{Z}^{g}} \exp \left\{2 \pi i\left({ }^{t} m s+\sum_{1 \leqq \alpha, \beta \leqq g} m_{\alpha} m_{\beta} \varrho_{\beta}\right)-\pi i \sum_{1 \leqq \alpha, \beta \leqq g} m_{\alpha} \varrho_{|\alpha-\beta|} m_{\beta}\right\} .
$$

Then $\Theta(s)$ satisfies the quasi-periodicity and evenness relations

$$
\begin{aligned}
\Theta(s+m) & =\Theta(s), & & \text { for } m \in \mathbb{Z}^{g}, \\
\Theta\left(s+\tau_{\beta}\right) & =e^{-2 \pi i\left(s_{\beta}+\varrho_{\beta}\right)} \Theta(s), & & \text { for } 1 \leqq \beta \leqq g, \\
\Theta(-s) & =\Theta(s) . & &
\end{aligned}
$$

The theta function $\vartheta\left[\begin{array}{l}\delta \\ v\end{array}\right]$ with rational characteristics $\delta, v$ of $\mathbb{Q}^{g}$ are defined by

$$
\vartheta\left[\begin{array}{l}
\delta \\
v
\end{array}\right](s, \tau)=e^{2 \pi i^{t} \delta(s+v)+\pi i^{t} \delta \tau \delta} \vartheta(s+\tau \delta+v, \tau),
$$

with the quasi-periodicity relation

$$
\vartheta\left[\begin{array}{l}
\delta \\
v
\end{array}\right](s+m, \tau)=e^{2 \pi i^{t} \delta m} \vartheta\left[\begin{array}{l}
\delta \\
v
\end{array}\right](s, \tau)
$$

$\vartheta\left[\begin{array}{l}\delta \\ v\end{array}\right](s+\tau m, \tau)=e^{-2 \pi i^{t} v m-2 \pi i^{t} m s-\pi i^{t} m \tau m} \vartheta\left[\begin{array}{l}\delta \\ v\end{array}\right](s, \tau), \quad$ for $m \in \mathbb{Z}^{g}$. 
We are going to describe the prime form of the Riemann surface $W$. Consider the divisor $D=\sum_{2 \leqq \beta \leqq g} \mathbf{b}_{\beta}$. Then the canonical bundle of $W$ equals to $\mathcal{O}_{W}[2 D]$. By Riemann-Roch Theorem and Lemma $5,|D|=$ a single divisor $D$. By the general theory of prime forms of Riemann surfaces (see e.g. [17]) implies the existence of a non-singular, odd, theta characteristic $\vartheta\left[\begin{array}{c}\bar{\delta} \\ \bar{v}\end{array}\right], \bar{\delta}, \bar{v} \in\left(\frac{1}{2} \mathbb{Z}\right)^{g} / \mathbb{Z}^{g}$, such that

$$
\vartheta\left[\begin{array}{c}
\bar{\delta} \\
\bar{v}
\end{array}\right]\left(\int_{\mathbf{w}^{\prime}}^{\mathbf{w}} \phi\right)=0 \Leftrightarrow\left\{\begin{array}{c}
\mathbf{w}^{\prime}=\mathbf{w} \\
\mathbf{w}^{\prime} \text { or } \mathbf{w}=\mathbf{b}_{\beta}, \text { for some } 2 \leqq \beta \leqq g
\end{array}\right.
$$

Then

$$
\zeta=\sum_{1 \leqq \beta \leqq g} \frac{\partial \vartheta\left[\begin{array}{l}
\bar{\delta} \\
\bar{v}
\end{array}\right]}{\partial s_{\beta}}(0) \phi_{\beta}
$$

is the unique holomorphic 1-form with $(\zeta)=2 D$. So $\sqrt{\zeta}$ is the section of $\mathcal{O}_{W}[D]$ and the prime form is given by

$$
E\left(\mathbf{w}_{0}, \mathbf{w}\right)=\frac{\vartheta\left[\begin{array}{l}
\bar{\delta} \\
\bar{v}
\end{array}\right]\left(\int_{\mathbf{w}_{0}}^{\mathbf{w}} \phi\right)}{\sqrt{\zeta(\mathbf{w})} \sqrt{\zeta\left(\mathbf{w}_{0}\right)}}, \text { for } \mathbf{w}_{0}, \mathbf{w} \in W .
$$

For a fixed point $\mathbf{w}_{0}$ of $W, E\left(\mathbf{w}_{0}, \mathbf{w}\right)$ defines a global section of $\mathcal{O}_{W}\left[\mathbf{w}_{0}\right]$ of the Riemann surface $W$. Therefore

$$
\frac{E\left(\mathbf{p}^{\prime}, \mathbf{w}\right)}{E(\mathbf{p}, \mathbf{w})}=\frac{\vartheta\left[\begin{array}{c}
\bar{\delta} \\
\bar{v}
\end{array}\right]\left(\int_{\mathbf{p}^{\prime}}^{\mathbf{w}} \phi\right)}{\vartheta\left[\begin{array}{l}
\bar{\delta} \\
\bar{v}
\end{array}\right]\left(\int_{\mathbf{p}}^{\mathbf{w}} \phi\right)}, \quad \mathbf{w} \in W,
$$

corresponds to a section of $\mathcal{O}_{W}\left[\mathbf{p}^{\prime}-\mathbf{p}\right]$. Consider the function of $\mathbb{C}^{g}$ :

$$
f(s)=\frac{\vartheta\left[\begin{array}{l}
\bar{\delta} \\
\bar{v}
\end{array}\right]\left(s-\frac{1}{2} \varrho-\frac{1}{2} \varepsilon, \tau\right)}{\vartheta\left[\begin{array}{l}
\bar{\delta} \\
\bar{v}
\end{array}\right]\left(s+\frac{1}{2} \varrho+\frac{1}{2} \varepsilon, \tau\right)} .
$$

By Proposition 9, $\frac{E\left(\mathbf{p}^{\prime}, \mathbf{w}\right)}{E(\mathbf{p}, \mathbf{w})}=f(s(\mathbf{w}))$, here $s(\mathbf{w})=\int_{\mathbf{0}}^{\mathbf{w}} \phi$. By the quasi-periodicity property of,$\left[\begin{array}{c}\bar{\delta} \\ \bar{v}\end{array}\right]$, we have

$$
\begin{aligned}
& f(s+m)=f(s), \\
& f(s+\tau m)=e^{2 \pi i^{t} m(\varrho+\varepsilon)} f(s), \quad \text { for } s \in \mathbb{C}^{g}, m \in \mathbb{Z}^{g} .
\end{aligned}
$$

Since

$$
\exp \left\{\frac{2 \pi i}{N} \sum_{1 \leqq \alpha \leqq g}\left(s_{\alpha}+\sum_{1 \leqq \beta \leqq g} \tau_{\alpha \beta} m_{\beta}\right)\right\}=e^{2 \pi i^{t} m \varrho} \exp \left\{\frac{2 \pi^{i}}{N} \sum_{1 \leqq \alpha \leqq g} s_{\alpha}\right\},
$$


the function

$$
\begin{aligned}
f_{\mathbf{p}^{\prime}, \mathbf{p}}(s) & =\exp \left\{\frac{-2 \pi i}{N} \sum_{\alpha=1}^{g} s_{\alpha}\right\} f(s) \\
& =\frac{\vartheta\left[\begin{array}{l}
\bar{\delta} \\
\bar{v}
\end{array}\right]\left(s-\frac{1}{2} \varrho-\frac{1}{2} \varepsilon, \tau\right)}{e^{2 \pi i / N \sum_{a} s_{a}} \vartheta\left[\begin{array}{l}
\bar{\delta} \\
\bar{v}
\end{array}\right]\left(s+\frac{1}{2} \varrho+\frac{1}{2} \varepsilon, \tau\right)}
\end{aligned}
$$

Satisfies the relation

$$
\begin{aligned}
f_{\mathbf{p}^{\prime}, \mathbf{p}}(s+m) & =e^{-2 \pi i^{t} m 1 / N} f_{\mathbf{p}^{\prime}, \mathbf{p}}(s), \\
f_{\mathbf{p}^{\prime}, \mathbf{p}}(s+\tau m) & =e^{2 \pi i^{t} m \varepsilon} f_{\mathbf{p}^{\prime}, \mathbf{p}}(s)
\end{aligned}
$$

for $s \in \mathbb{C}^{g}, m \in \mathbb{Z}^{g} ;$ here $\frac{1}{N}=\sum_{1 \leqq \beta \leqq g} e_{\beta}$. The restriction of $f_{\mathbf{p}^{\prime}, \mathbf{p}}$ on $W$ [via (29) for $\star=\mathbf{0}]$ can be regarded as a section in $\Gamma\left(W, \mathcal{O}_{W}\left(\mathbf{p}^{\prime}-\mathbf{p}\right)\right)$ with the divisor $\operatorname{div}\left(f_{\mathbf{p}^{\prime}, \mathbf{p}}\right)=\mathbf{p}^{\prime}-\mathbf{p}$. Similarly, the functions of $\mathbb{C}^{g}$ defined by

$$
\begin{gathered}
f_{\mathbf{q}, \mathbf{p}}(s)=\frac{\vartheta\left[\begin{array}{l}
\bar{\delta} \\
\bar{v}
\end{array}\right]\left(s+\frac{1}{2} \varrho-\frac{1}{2} \varepsilon, \tau\right)}{\vartheta\left[\begin{array}{l}
\bar{\delta} \\
\bar{v}
\end{array}\right]\left(s+\frac{1}{2} \varrho+\frac{1}{2} \varepsilon, \tau\right)} \\
f_{\mathbf{q}^{\prime}, \mathbf{p}}(s)=\frac{\vartheta\left[\begin{array}{l}
\bar{\delta} \\
\bar{v}
\end{array}\right]\left(s-\frac{1}{2} \varrho+\frac{1}{2} \varepsilon, \tau\right)}{e^{2 \pi i / N \sum_{1 \leqq a \leqq g} s_{a}} \vartheta\left[\begin{array}{l}
\bar{\delta} \\
\bar{v}
\end{array}\right]\left(s+\frac{1}{2} \varrho+\frac{1}{2} \varepsilon, \tau\right)}
\end{gathered}
$$

satisfy the relations

$$
\begin{gathered}
f_{\mathbf{q}, \mathbf{p}}(s+m)=f_{\mathbf{q}, \mathbf{p}}(s), \quad f_{\mathbf{q}, \mathbf{p}}(s+\tau m)=e^{2 \pi i^{t} m \varepsilon} f_{\mathbf{q}, \mathbf{p}}(s), \\
f_{\mathbf{q}^{\prime}, \mathbf{p}}(s+m)=e^{-2 \pi i^{t} m 1 / N} f_{\mathbf{q}^{\prime}, \mathbf{p}}(s), \quad f_{\mathbf{q}^{\prime}, \mathbf{p}}(s+\tau m)=f_{\mathbf{q}^{\prime}, \mathbf{p}}(s),
\end{gathered}
$$

for $s \in \mathbb{C}^{g}, m \in \mathbb{Z}^{g}$. The restrictions of $f_{\mathbf{q}, \mathbf{p}}, f_{\mathbf{q}^{\prime}, \mathbf{p}}$ on $W$ are the section in $\Gamma\left(W, \mathcal{O}_{W}(\mathbf{q}-\mathbf{p})\right), \Gamma\left(W, \mathcal{O}_{W}\left(\mathbf{q}^{\prime}-\mathbf{p}\right)\right)$ respectively, with $\operatorname{div}\left(f_{\mathbf{q}, \mathbf{p}}\right)=\mathbf{q}-\mathbf{p}$, $\operatorname{div}\left(f_{\mathbf{q}^{\prime}, \mathbf{p}}\right)=\mathbf{q}^{\prime}-\mathbf{p}$. Note that the phase factors in the quasi-periodicity relations of $f_{\mathbf{p}^{\prime}, \mathbf{p}}, f_{\mathbf{q}, \mathbf{p}}, f_{\mathbf{q}^{\prime}, \mathbf{p}}$ are the $N^{\text {th }}$ roots of unity. These functions are closely connected to the variables $a, b, c, d$ of the $N^{2}$-cover $\mathfrak{M}$ of $W$ in Sect. 4 . Since the $N^{\text {th }}$ power of $f_{\mathbf{p}^{\prime}, \mathbf{p}}, f_{\mathbf{q}, \mathbf{p}}, f_{\mathbf{q}^{\prime}, \mathbf{p}}$ gives the rational function on $W$, the situation is much simpler for the purpose of the expression of the parameter $r$ of $W$.

Consider first the case when $r$ equals to $k^{\prime}$. As before, let $\mathfrak{W}$ be the curve defined in (21) with the homogeneous coordinates $a, b, c, d$. The above $\mathbf{p}, \mathbf{p}^{\prime}, \mathbf{q}, \mathbf{q}^{\prime}$ are now the $\mathbf{u}, \mathbf{u}^{\prime}, \mathbf{v}, \mathbf{v}^{\prime}$ in Proposition 7. As the rational functions of $W, f_{\mathbf{v}, \mathbf{u}}^{N}, f_{\mathbf{v}^{\prime}, \mathbf{u}}^{N}$, $f_{\mathbf{u}^{\prime}, \mathbf{u}}^{N}$, equal to $\frac{a^{N}}{d^{N}}, \frac{b^{N}}{d^{N}}, \frac{c^{N}}{d^{N}}$ up to some constants, i.e. 


$$
\begin{aligned}
& a^{N}: b^{N}: c^{N}: d^{N}=A e^{2 \pi i \Sigma_{a} s_{a}} \vartheta\left[\begin{array}{l}
\bar{\delta} \\
\bar{v}
\end{array}\right]\left(s+\frac{1}{2} \varrho-\frac{1}{2} \varepsilon, \tau\right)^{N} \\
& : B \vartheta\left[\begin{array}{l}
\bar{\delta} \\
\bar{v}
\end{array}\right]\left(s-\frac{1}{2} \varrho+\frac{1}{2} \varepsilon, \tau\right)^{N} \\
& : C \vartheta\left[\begin{array}{l}
\bar{\delta} \\
\bar{v}
\end{array}\right]\left(s-\frac{1}{2} \varrho-\frac{1}{2} \varepsilon, \tau\right)^{N} \\
& : e^{2 \pi i \sum_{a} s_{a}} \vartheta\left[\begin{array}{l}
\bar{\delta} \\
\bar{v}
\end{array}\right]\left(s+\frac{1}{2} \varrho+\frac{1}{2} \varepsilon, \tau\right)^{N} \text {, }
\end{aligned}
$$

here $s$ is the uniformizing coordinate of elements of $W$, and $A, B, C$ are constants. The hyperelliptic involution $\sigma$ of $W$ induces the automorphism $\mathfrak{J}$ of $\mathfrak{M}$ [defined in (23)], which corresponds the map of $\mathbb{C}^{g}, s \leadsto-s$. The relations

$$
\mathfrak{J}^{*}\left(\frac{a^{N}}{d^{N}}\right)=\frac{b^{N}}{c^{N}}, \quad \mathfrak{J}^{*}\left(\frac{c^{N}}{d^{N}}\right)=\frac{d^{N}}{c^{N}},
$$

imply $B=A C, C^{2}=1$. For $s=$ the origin, it corresponds to the element $\mathbf{o}$ of $W$ with the coordinate $(t, \lambda)$ [defined in (4)] $=\left(\left(\frac{1+k^{\prime}}{1-k^{\prime}}\right)^{1 / N},-1\right)$. By (19) (20), the
constants $A, B, C$ must satisfy

$$
A=B, \quad C=1 .
$$

By (21), we have the relation

$$
\begin{aligned}
& k A e^{2 \pi i \sum_{a} s_{a} \vartheta}\left[\begin{array}{l}
\bar{\delta} \\
\bar{v}
\end{array}\right]\left(s+\frac{1}{2} \varrho-\frac{1}{2} \varepsilon, \tau\right)^{N}+k^{\prime} \vartheta\left[\begin{array}{l}
\bar{\delta} \\
\bar{v}
\end{array}\right]\left(s-\frac{1}{2} \varrho-\frac{1}{2} \varepsilon, \tau\right)^{N} \\
& =e^{2 \pi i \sum_{a} s_{a} \vartheta}\left[\begin{array}{l}
\bar{\delta} \\
\bar{v}
\end{array}\right]\left(s+\frac{1}{2} \varrho+\frac{1}{2} \varepsilon, \tau\right)^{N} .
\end{aligned}
$$

Substituting $s=\frac{-1}{2} \varrho+\frac{1}{2} \varepsilon$, by (31) we obtain

$$
k^{\prime}=\frac{-e^{-\pi i\left(\varrho_{1}+\ldots+\varrho_{g}\right)} \vartheta\left[\begin{array}{l}
\bar{\delta} \\
\bar{v}
\end{array}\right](\varepsilon, \tau)^{N}}{\vartheta\left[\begin{array}{l}
\bar{\delta} \\
\bar{v}
\end{array}\right](\varrho, \tau)^{N}} .
$$

When $r=i \frac{k^{\prime}}{k}$, the CP $N$-curve is the curve $W_{r}$ of (9) in Sect. 3. Its hyperelliptic involution is induced by the automorphism $\mathfrak{I}_{r}$ of $\mathfrak{M}$ by Proposition 6 (ii). Let $\tau_{r}$ be the period for $W_{r}$. By Proposition 8,

$$
\begin{aligned}
& a^{N}: b^{N}: c^{N}: d^{N}=A_{r} e^{2 \pi i \Sigma_{a} s_{a}} \vartheta\left[\begin{array}{l}
\bar{\delta} \\
\bar{v}
\end{array}\right]\left(s+\frac{1}{2} \varrho_{r}-\frac{1}{2} \varepsilon, \tau_{r}\right)^{N}: B_{r} \vartheta\left[\begin{array}{l}
\bar{\delta} \\
\bar{v}
\end{array}\right]\left(s-\frac{1}{2} \varrho_{r}-\frac{1}{2} \varepsilon, \tau_{r}\right)^{N} \\
& : C_{r} \vartheta\left[\begin{array}{l}
\bar{\delta} \\
\bar{v}
\end{array}\right]\left(s-\frac{1}{2} \varrho_{r}+\frac{1}{2} \varepsilon, \tau_{r}\right)^{N}: e^{2 \pi i \Sigma_{a} s_{a} \vartheta}\left[\begin{array}{l}
\bar{\delta} \\
\bar{v}
\end{array}\right]\left(s+\frac{1}{2} \varrho_{r}+\frac{1}{2} \varepsilon, \tau_{r}\right)^{N},
\end{aligned}
$$

with $B_{r}=A_{r} C_{r},\left(B_{r}\right)^{2}=-1$. By the definition of the element $\mathbf{o}$ of $W_{r}$, together with the description of the function $f^{\prime}$ in the remark (i) of Proposition 4 , the $\lambda$ - 
value of $\mathbf{o}$ equals to $k^{\prime}+i k$. By the relations (17) (20), $\frac{b^{N}}{d^{N}}=\frac{\lambda^{-1}-k^{\prime}}{k}$, hence $B_{r}=(-1)^{g} i$. By $(22)$,

$$
\begin{gathered}
A_{r} e^{2 \pi i \sum_{a} s_{a} \vartheta}\left[\begin{array}{l}
\bar{\delta} \\
\bar{v}
\end{array}\right]\left(s+\frac{1}{2} \varrho_{r}-\frac{1}{2} \varepsilon, \tau_{r}\right)^{N}+k^{\prime} i(-1)^{g} \vartheta\left[\begin{array}{l}
\bar{\delta} \\
\bar{v}
\end{array}\right]\left(s-\frac{1}{2} \varrho_{r}-\frac{1}{2} \varepsilon, \tau_{r}\right)^{N} \\
=k e^{2 \pi i \sum_{a} s_{a}} \vartheta\left[\begin{array}{l}
\bar{\delta} \\
\bar{v}
\end{array}\right]\left(s+\frac{1}{2} \varrho_{r}+\frac{1}{2} \varepsilon, \tau_{r}\right)^{N} .
\end{gathered}
$$

Hence

$$
\frac{i k^{\prime}}{k}=\frac{(-1)^{N} e^{-\pi i\left(\varrho_{r 1}+\ldots+\varrho_{r g}\right)} \vartheta\left[\begin{array}{l}
\bar{\delta} \\
\bar{v}
\end{array}\right]\left(\varepsilon, \tau_{r}\right)^{N}}{\vartheta\left[\begin{array}{l}
\bar{\delta} \\
\bar{v}
\end{array}\right]\left(\varrho_{r}, \tau_{r}\right)^{N}}
$$

For the case $r=k$, let $\tau_{l}$ be the period of $W_{N, k}\left(\simeq W_{l}\right.$ in (11)). Similarly, we have $\begin{aligned} a^{N}: b^{N}: c^{N}: d^{N}= & (-1)^{g} \vartheta\left[\begin{array}{l}\bar{\delta} \\ \bar{v}\end{array}\right]\left(s-\frac{1}{2} \varrho_{l}-\frac{1}{2} \varepsilon, \tau_{l}\right)^{N}: B_{l} e^{2 \pi i \Sigma_{a} s_{a} \vartheta}\left[\begin{array}{l}\bar{\delta} \\ \bar{v}\end{array}\right]\left(s+\frac{1}{2} \varrho_{l}-\frac{1}{2} \varepsilon, \tau_{l}\right)^{N} \\ & :(-1)^{g} B_{l} \vartheta\left[\begin{array}{l}\bar{\delta} \\ \bar{v}\end{array}\right]\left(s-\frac{1}{2} \varrho_{l}+\frac{1}{2} \varepsilon, \tau_{l}\right)^{N}: e^{2 \pi i \sum_{a} s_{a} \vartheta}\left[\begin{array}{l}\bar{\delta} \\ \bar{v}\end{array}\right]\left(s+\frac{1}{2} \varrho_{l}+\frac{1}{2} \varepsilon, \tau_{l}\right)^{N},\end{aligned}$

and

$$
\begin{aligned}
& (-1)^{g} \vartheta\left[\begin{array}{l}
\bar{\delta} \\
\bar{v}
\end{array}\right]\left(s-\frac{1}{2} \varrho_{l}-\frac{1}{2} \varepsilon, \tau_{l}\right)^{N}+k^{\prime} B_{l} e^{2 \pi i \Sigma_{a} s_{a} \vartheta}\left[\begin{array}{l}
\bar{\delta} \\
\bar{v}
\end{array}\right]\left(s+\frac{1}{2} \varrho_{l}-\frac{1}{2} \varepsilon, \tau_{l}\right)^{N} \\
& =k e^{2 \pi i \sum_{a} s_{a}} \vartheta\left[\begin{array}{l}
\bar{\delta} \\
\bar{v}
\end{array}\right]\left(s+\frac{1}{2} \varrho_{l}+\frac{1}{2} \varepsilon, \tau_{l}\right)^{N},
\end{aligned}
$$

hence

$$
k=\frac{(-1)^{N} \vartheta\left[\begin{array}{l}
\bar{\delta} \\
\bar{v}
\end{array}\right]\left(\varrho_{l}, \tau_{l}\right)^{N}}{e^{-\pi i\left(\varrho_{l 1}+\ldots+\varrho_{l g}\right)} \vartheta\left[\begin{array}{l}
\bar{\delta} \\
\bar{v}
\end{array}\right]\left(\varepsilon, \tau_{l}\right)^{N}}
$$

Using the identities obtained by Sonya Kowalevski [14], Baxter found the explicit expression of the above $\bar{\delta}, \bar{v}$, and they are given by

$$
\bar{\delta}=\frac{1}{2}\left(\begin{array}{c}
1 \\
0 \\
\vdots \\
0 \\
0
\end{array}\right), \quad \bar{v}=-\frac{1}{2}\left(\begin{array}{c}
1 \\
1 \\
\vdots \\
1 \\
1
\end{array}\right)
$$

(The above $\vartheta\left[\begin{array}{l}\bar{\delta} \\ \bar{v}\end{array}\right](\mathrm{s}, \tau)$ is the function $\Theta\{s\}_{1}$ of [6].) Therefore we have obtained the following conclusion.

Theorem. Let $\tau, \tau_{r}, \tau_{l}$ be the period for the CP $N$-curves $W_{N, k^{\prime}}, W_{N, i k^{\prime} \mid k}, W_{N, k}$ respectively. Then the relations between $k^{\prime}, i \frac{k^{\prime}}{k}, k$ and $\tau, \tau_{r}, \tau_{l}$ are given by (33), (34), (35) and (36). 


\section{References}

1. Albertini, G., McCoy, B.M., Perk, J.H.H.: Eigenvalue spectrum of the superintegrable chiral Potts model. Adv. Stud. Pure Math. 19, 1-55 (1989)

2. Albertini, G., McCoy, B. M., Perk, J.H. H.: Commensurate-incommensurate transition in the ground state of the superintegrable chiral Potts model. Phys. Lett. A135, 159-166 (1989)

3. Albertini, G., McCoy, B. M., Perk, J.H.H.: Level crossing transitions and the massless phases of the superintegrable chiral Potts chain. Phys. Lett. A139, 204-212 (1989)

4. Albertini, G., McCoy, B. M., Perk, J. H. H., Tang, S.: Excitation spectrum and order parameter for the integrable $N$-state chiral Potts model. Nucl. Phys. B314, 741-763 (1989)

5. Au-Yang, H., Perk, J.H. H.: Onsager's star-triangle equation: Master key to integrability. Adv. Stud. Pure Math. 19, 57-94 (1989)

6. Baxter, R. J.: Hyperelliptic function parametrization for the chiral Potts model. preprint

7. Baxter, R. J.: Corner transfer matrices of the chiral Potts model, preprint

8. Baxter, R. J.: Solving models in statistical mechanics. Adv. Stud. Pure Math. 19, 95-116 (1989)

9. Baxter, R. J.: The superintegrable chiral Potts model. Phys. Lett. A 133, 185-189 (1988)

10. Baxter, R. J.: Chiral Potts model: eigenvalues of the transfer matrix. Phys. Lett. A146, 110114 (1990)

11. Baxter, R. J., Bazhanov, V.V., Perk, J.H.H.: Functional relations for transfer matrices of the chiral Potts model. Int. J. Mod. Phys. B4, 803 (1990)

12. Baxter, R. J., Perk, J. H. H., Au-Yang, H.: New solutions of the star-triangle relations for the chiral Potts model. Phys. Lett. A128, 138-142 (1988)

13. Bazhanov, V.V., Stroganov, Yu. G.: Chiral Potts model as a decendant of the six-vertex model. J. Stat. Phys. 59, 799-817 (1990)

14. Kowalevski, S.: Sur le probleme de la rotation d'un corps solide autour d'un point fixe. Acta Math. 12, 177-232 (1889)

15. McCoy, B. M.: The chiral Potts model: From physics to mathematics and back, Lecture in Okayama conference, Japan (1990)

16. McCoy, B. M., Roan, S.-S.: Excitation spectrum and phase structure of the chiral Potts model. Phys. Lett. A 150, 347-354 (1990)

17. Mumford, D.: Tata lectures on theta I, II, Progress in Mathematics, Vol. 28 and Vol. 43. Basel: Birkhäuser

Communicated by H. Araki 